\title{
Analytic Comparison of Suggested Configurations for Automatic Mail Sorting Equipment ${ }^{1}$
}

\author{
B. K. Bender and A. J. Goldman
}

(June 5, 1959)

\begin{abstract}
Analytic methods are developed to aid in determining the equipment configuration which achieves sorting of outgoing mail at a given (required) rate at least cost. The techniques are applied to a specific numerical problem; several of the suggested configurations are quickly eliminated, and a "hybrid" of two of the proposed configurations is found which comes within four percent of optimum (if a certain pair of parameters is chosen correctly).
\end{abstract}

\section{Introduction}

A number of plausible configurations for automatic mail sorting equipment have been suggested. In this report methods for the comparative study of such configurations are developed, with the object of determining the configuration which achieves sorting of mail at a given (required) rate at least cost.

The solution to this "optimization" problem of course depends upon the distribution (by destinations) and volume of mail at the sorting installation, and also on the operating parameters and costs associated with the various components of the sorting system. Therefore, in order to present our methods in the most comprehensible way (i.e., in the context of a specific numerical application), it was necessary to work with a definite set of realistic "input" data. These inputs are described in sections 3 to 6 and appendix A. We wish to emphasize that we work with these particular data only to illustrate the methods employed; the reader who follows out the calculations for this specific case should have little trouble carrying out the corresponding calculations for other data.

The results of the analyses are summarized in section 2 . In brief, we are able $(i)$ to rule out several of the proposed configurations, (ii) to show that one of the proposed configurations will (if a certain parameter is choosen correctly) come within 8 per cent of optimum, and (iii) to exhibit a "hybrid" of two of the proposed configurations which (if a certain pair of parameters is chosen correctly) will come within 4 percent of optimum.

We also determine how accurately the parameters in (ii) and (iii) must be chosen. In view of (iii) analysis of more complicated configurations did not seem worthwhile.

For a comparative study, it is necessary only to consider costs which vary appreciably from one configuration to another. Our analysis is set up so that these variable costs are all absorbed into costs associated with $(a)$ the devices which inject mail into the sorting system (we will generally use the

1 The preparation of this paper was sponsored by the Post Office Department Office of Research and Engineering. term loading complex for such a device, instead of the longer "distributor loader complex"), (b) the receptacles (bins) in which the mail ends after passing through each stage of the system, and $(c)$ the operation (sweeping) of removing the mail from these bins.

A natural method of determining the cost of any proposed configuration, therefore, is to determine the numbers of loading complexes, bins, and sweepers involved, and then to multiply each of these numbers by the appropriate cost coefficient and add up the resulting partial costs. We might call this the add up method; it is described in section 7 and at the end of section 8 . We have also developed a follow through method based on the idea of following an individual letter through the sorting system, charging it an appropriate fraction of the cost of each loading complex through which it passes, each loading complex by which it passes (and thus prevents from operating at maximum rate), each bin it occupies, and each sweep it receives. The follow through method is not strictly applicable to some configurations (though it does seem to provide initial approximations which may shorten some of the work of the "add up" method); its main advantage is that it permits a much closer estimation ${ }^{2}$ of the theoretical minimum cost described in the next paragraph.

An important step in the anslysis is the derivation of a theoretical minimum cost (more precisely, a "lower bound") which is independent of the configuration. This cost is minimum in the sense that "any actual configuration must cost at least this much" and is theoretical. in the sense that "no actual configuration can cost quite this little"; it therefore provides a yardstick against which the costs of specific configurations can be measured, the deviation from optimality of a configuration can be assessed, and the permissibility of plausible approximations can be checked. A rough estimate of such a minimum cost is given in section 8 (without using the specific mail distribution); in section 9 the "follow through" method is employed to derive a significantly more accurate result. ${ }^{2}$

\footnotetext{
2 The importance of this is discussed in section 8 .
} 
The detailed analyses of the configurations studied are given in sections 10 to 14 and appendixes B and C. We hope that our rather full exposition of this material will prove helpful to the reader faced with the problem of carrying out similar analyses.

\section{Summary of Results}

From our specific numerical results (which are valid only for the particular data and assumptions used in our analysis) we can infer certain qualitative results, believed to be valid for post-offices $(i)$ for which the sorting system must be able to handle about $1,000,000$ letters/hr or more and (ii) in which local mail is removed before the outgoing sort.

Of these two types of results, the qualitative ones are more informative, and so we list them first:

(a) The simplex scheme and Christmas Tree scheme ${ }^{3}$ can be eliminated from further consideration.

(b) The residue scheme (if properly chosen) is quite good; some multiple input schemes may possibly be competitive.

(c) A promising approach is to combine the basic ideas of the residue and multiple input schemes; the resulting "hybrid" scheme has lower cost than either of the two original schemes if the relevant parameters are correctly chosen.

We turn now to the specific numerical results. The "inputs" leading to these results are given in detail in sections $3-6$ and appendix $A$; at this point we only add to (i) and (ii) above the facts that (iii) only costs which vary between different equipment configurations were considered, $(i v)$ the use of automatic transfer equipment was not considered, and $(v)$ the possibility of memory-sharing between oading complexes was not considered.

Condition (iii) is a natural one, since we are primarily interested in comparing different configurations. Auxiliary calculations (not given in this paper) show that the qualitative results reported above do not depend on $(i v)$ and $(v)$.

As noted in section 1, a "theoretical minimum cost" is used as a yardstick against which the desirability of any proposed configuration can be measured. For this purpose we use the ratio

$\mathrm{R}=\frac{\text { (Cost of proposed config.) }- \text { (Theoretical minimum) }}{\text { (Theoretical }}$

"Near-optimal" configurations are those with small values of $R$. In the notation of the body of the report, (2.1) becomes

$$
R=\left(C-C_{\min }^{*}\right) / C_{\min }^{*} .
$$

Our specific numerical results are as follows:

(a) The theoretical minimum (yearly variable) cost is about $\$ 2,050,000$.

(b) For the simplex scheme, $R$ is about 160 percent;

\footnotetext{
3 These schemes are described as they arise later in the report
}

i.e., the scheme costs about 2.6 times the theoretical minimum cost.

(c) For the Christmas Tree scheme, $R$ is at least 69 percent; i.e., the scheme costs at least 1.69 times the theoretical minimum cost.

(d) For the best residue scheme, $R$ is about 8 percent.

(e) There are a great many ways in which the basic ideas of the residue and multiple input schemes can be combined. An analysis of the full range of possibilities would be beyond the scope of our study. We have, however, examined a relatively simple class of systems of this type, in which each subsystem involves only two loading complexes in series; the optimal configuration within this class has $R$ about 4 percent, which ( $i$ ) compares favorably with all other configurations studied, and (ii) indicates that investigation of more complicated systems would not be worthwhile.

(f) All multiple input schemes with more than four subsystems (i.e., more than four series of loading complexes) have $R$ at least 8 percent (but probably substantially more); multiple input schemes with four subsystems have $R$ at least 4 percent (but probably substantially more). We do not have a complete proof that multiple input schemes with fewer than four subsystems have excessively high values of $R$ (in comparison with $(d)$ and $(e)$ ), but strongly believe that this is the case. Each subsystem of such a configuration would involve nine or more loading complexes in series.

\section{Equipment Cost Data}

Only those equipment costs which appeared likely to vary appreciably from one configuration to another were considered. The cost of coding the letters, for example, was neglected on the grounds that in a code sort system, all letters to be sorted must be coded once and only once, regardless of how many readings or sorts they undergo.

The two pieces of equipment whose costs were considered variable are the loading complexes and the modules of bins. Hereafter, the cost of bins for the sorted mail will be considered to include the costs of the corresponding conveyor, cart pockets, etc.

The following tentative cost estimates were given by a representative of the manufacturer:

$\$ 2,000 /$ module of 30 bins,

$\$ 125,000 /$ first loading complex of a sorter,

$\$ 50,000 /$ each of the next 4 loading complexes of a sorter,

$\$ 10,000 /$ pair of end-pieces.

Because (a) total equipment costs are somewhat lower than total personnel costs (see the three summands of (8.6), for example) and $(b)$ the estimates (3.1) to (3.4) are only rough ones, it seemed permissible to be rather loose in our treatment of equip- 
ment costs. First, the end-piece costs are small relative to other costs, and are difficult to handle because of the way in which the number of endpieces depends on the particular placement of equipment. For these reasons, the end-piece costs have been neglected altogether. Second, there was apparently some doubt as to whether the extra loading complexes of a multi-input sorter can actually be used in the manner assumed in the estimate (3.3); for this reason we have disregarded (3.3) and taken the cost of every loading complex to be $\$ 125,000$. Our numerical work for specific sorting schemes is fairly sensitive to these decisions, but the results of comparing different schemes are not.

In summary, the equipment costs actually used in what follows are

$\$ 2,000 /$ module of 30 bins

$\$ 125,000 /$ loading complex

These are initial costs; yearly costs are based on a 10-yr amortization period.

\section{Space Cost Data}

The only space requirements taken into account ${ }^{4}$ were those for the loading complexes themselves, for bins, and for the sweepers' work area around them:

$8.75 \mathrm{ft}=$ length of a loading complex.

$8.75 \mathrm{ft}=$ length of a module of 30 bins.

$11.25 \mathrm{ft}=$ width of a module, including sweepers' work space

Using the tentative cost figure

$$
\$ 2.20 / \mathrm{ft}^{2} / \mathrm{yr}
$$

suggested by L. Allison (NBS Electronic Instrumentation Section) we obtain $(8.75) \times(11.25) \times(2.2)$ $\approx 220$, or

$$
\$ 220 \text { /loading complex/yr }
$$

$\$ 220 /$ module of $30 \mathrm{bins} / \mathrm{yr}$.

It is convenient to combine space and equipment costs. From (3.6) and (4.5), remembering the 10 -yr amortization applying to (3.6), we have

$\$ 12,500+\$ 220 \approx \$ 12,700 /$ loading complex/yr.

Similarly, from (3.5) and (4.6) we have $\$ 200+\$ 220$ $=\$ 420 /$ module of $30 \mathrm{bins} / \mathrm{yr}$, so that we have

$$
\$ 14 / \mathrm{bin} / \mathrm{yr} \text {. }
$$

Formulas (4.7) and (4.8) are the ones used in our analysis.

\section{Personnel Cost Data}

The personnel which appears to vary appreciably from one configuration to another is composed of

\footnotetext{
${ }_{4}^{4}$ These gave the costs which appeared likely to vary appreciably from one configuration to another.
}

sweepers and pouchers. The tentative data used below were suggested by L. Allison.

Sweep operators remove sorted letters from the bins and either tie them out or place them in trays preceding a further sort. We take

60 letters $=$ number of letters a sweeper can be expected to handle in one sweep,

180 sweeps $/ \mathrm{hr}=$ working rate expected of a sweeper.

It is assumed that the sweeping is carried on in such away that:

Each bin is swept at least once per hour,

if more than 60 letters $/ \mathrm{hr}$ are expected in a particular bin, then (subject to over-rule by (5.3)) the bin is not swept when it contains fewer than 60 letters, and

no more than 20 sweeps (i.e., 1,200 letters)/ $\mathrm{hr}$ is expected for any one bin.

The rules (5.1) to (5.5) permit computation of the number of sweepers required, if it is known how many letters/hr are expected in each bin. This quantity can in turn be computed (as will be done in later sections) for any particular sorting scheme and mail distribution.

Pouching operators toss tied bundles of mail into pouches; their working rate is

$$
540 \text { sweeps pouched/hr. }
$$

Comparing (5.2) and (5.6), we see that, for sorts which are immediately followed by pouching,

number of pouchers $=1 / 3 \times$ (number of sweepers).

As for sorts which are not followed by pouching (i.e., primary sorts to be followed by secondary sorts), we observe that the additional work involved in handling the trays and feeding the loading complexes for a secondary sort appears to be of the same order of magnitude as that of pouching a similar quantity of mail. Therefore, since we suppose the transfer between sorts to be nonautomatic, we will take the cost of the personnel needed for this transfer to be equal to that of a number of fictitious pouchers given by (5.7). ${ }^{5}$

The average salary figure used is

$$
\$ 11,000 / \text { man-position/yr, }
$$

where a "man-position" requires more than one person because of the several shifts worked and the 7 -day week involved.

\section{Mail Distribution}

The particular mail distribution assumed in our numerical work is a hypothetical one obtained by

\footnotetext{
${ }^{5}$ In contrast, the personnel required for initial loading on the primary sort is nearly independent of the equipment configuration, and so is not considered.
} 
modifying data of outgoing mail from Los Angeles. ${ }^{6}$ It was decided that for a code sort machine, at least, all local, postage due, uncancelled mail, etc., could be removed at the coding station. The mail listed as go backs and residue (about 4\%) was excluded from sorter input because of the difficulty of distributing it properly by destination. The mail distribution to the sorting system, therefore, consists approximately of what is left after these deletions, with the percentage of mail to each destination upgraded to bring the total to 100 percent, and the amount of mail to each destination upgraded to bring the total to

$$
1,000,000 \text { letters } / \text { hr. }
$$

Our distribution involves

$$
\text { 1,600 destinations. }
$$

The details of the distribution are given in appendix A; they involve some inconsequential grouping in the "tail" of the distribution.

\section{Cost Formula}

The three variables in the cost formula are

$L=$ number of loading complexes,

$B=$ number of bins, and

$S=$ number of sweeper man-positions.

The values of these variables can be found for any particular mail distribution and specified arrangement of equipment.

The total yearly cost $C$ is given by

where

$$
C=C_{e}+C_{s}+C_{p},
$$

$C_{e}=$ yearly equipment cost,

$C_{s}=$ yearly space cost, and

$C_{p}=$ yearly personnel cost.

Using (4.7) and (4.8), we have

$$
C_{e}+C_{s}=12,700 L+14 B ;
$$

using (5.7) and (5.8) we have

$$
C_{p}=11,000\left(S+\frac{1}{3} S\right) \approx 14,670 S .
$$

The cost formula we will use is therefore

$$
C=12,700 L+14 B+14,670 S
$$

where $L, B$, and $S$ are defined above and

$$
C=\text { variable dollar cost } / \mathrm{yr} \text {. }
$$

\footnotetext{
${ }^{6}$ N. C. Severo and A. E. Newman, A statistical chain ratio method for deter-
} mining the distribution of mail by destination (to be published).

\section{General Minimum Cost Estimate}

We will first derive a theoretical minimum cost estimate which is general in the sense that it does not depend on the particular distribution by destinations of mail, but only on the volume of mail; i.e., on the fact that the sorting system must be able to handle

$$
1,000,000 \text { letters/hr. }
$$

Using this and the fact that the maximum input rate is

$$
\text { 36,000 letters/loading complex/hr, }
$$

we see that at least $1,000,000 / 36,000=27^{+}$loading complexes are required; since $L$ is an integer,

$$
L \geq 28 \text {. }
$$

By (5.5) each bin accounts for at most 1,200 letters/ hr, so that in view of (8.1) the number of bins required is at least

$$
1,000,000 / 1,200=833^{+} \text {; }
$$

since bins come in 30-bin modules, $B$ must be a multiple of 30 , and so

$$
B \geq 840 .
$$

By (5.1) each stack of letters contains at most 60 letters, so there must be at least

$$
1,000,000 / 60 \quad \text { stacks } / \mathrm{hr},
$$

and thus in view of $(5.2)$ there must be at least

$$
(1,000,000 / 60) / 180=92^{+} \text {sweepers }
$$

$$
\text { thus } \quad S \geq 93 \text {. }
$$

We now apply (8.3), (8.4), and (8.5) to (7.4), obtaining

$$
C \geq(12,700) \times(28)+(14) \times(840)+(14,670) \times(93) .
$$

or, rounding off,

$$
C \geq 356,000+12,000+1,364,000 \approx 1,732,000 .
$$

Thus we have proved that $\$ 1,732,000$ is an (approximate) minimum variable yearly total cost for equipment, space, and personnel. The three summands in (8.6) refer, respectively, to costs associated with loading complexes, bins, and personnel. We emphasize that this is a theoretical minimum cost; no actual system can cost this little.

Next we will be a little more realistic (and a little less general), and use the following one fact about the particular mail distribution with which we deal: Each of the 700 least frequent destinations receive 60 or fewer letters/hr. On the one hand, they re- 
quire at least $700 \mathrm{sweeps} / \mathrm{hr}$ and therefore yield at least 700 stacks $/ \mathrm{hr}$. On the other hand, these 700 destinations account for about 1.8 percent of the total mail, and thus for 18,000 letters $/ \mathrm{hr}$; if these letters were arranged into stacks of size 60 (as was assumed in deriving (8.5)), then

$$
18,000 / 60=300 \mathrm{stacks} / \mathrm{hr} \text {, }
$$

rather than 700 , would result. Thus the argument leading to (8.5) underestimates the minimum possible number of stacks $/$ hr by $700-300=400$, and thus (using (5.2)) underestimates $S$ by $400 / 180 \approx 2$ sweepers. (8.5) should be replaced by

$$
S \geq 95
$$

and the corresponding modification of (8.6), after rounding, is

$$
C \geq 1,761,000
$$

so that we have a minimum cost estimate of

$$
C_{\min }=\$ 1,761,000 \text {. }
$$

The estimate (8.8) is still too "general" to use as a yardstick, and we shall use instead a "detailed minimum cost estimate" (based on the detailed properties of the specific mail distribution) which will be derived in section 9. This care in choosing a yardstick might seen unnecessary, since a "yardstick" is only a unit of measurement whose choice cannot affect which of two proposed sorting systems appears less costly. The choice of yardstick does, however, affect our decisions as to what constitutes a significant difference in cost (either between two systems being compared or between a single system and a hypothetical "minimum-cost" system), and also as to what constitutes an allowable error in making simplifying approximations.

We shall use (8.8) primarily as an aid in calculating the costs of the various systems studied. If, using (8.3), (8.4), and (8.7), we define

$$
\begin{aligned}
& \Delta L=L-28 \\
& \Delta B=B-840 \\
& \Delta S=S--95,
\end{aligned}
$$

then it turns out that a convenient way to calculate the cost $C$ of a system is $(i)$ to find these numbers $\Delta L, \Delta B$, and $\Delta S$ of "extra" loading complexes, bins, and sweepers, (ii) to calculate an "extra cost,"

$$
\Delta C=C-C_{\min },
$$

by $\Delta C=(12,700) \Delta L+14 \Delta B+(14,670) \Delta S$

(this formula follows from (7.4)), and (iii) to find $C$ by

$$
C=C_{\min }+(\Delta C) .
$$

This constitutes the "add up" method mentioned in section 1.

\section{Detailed Minimum Cost Estimate}

Our main goal in this section is to derive a minimum cost estimate which makes detailed use of the particular mail distribution we are studying. The methods used in this derivation will also turn out to be helpful in the analysis of some of the systems considered later in the report. Reading the first paragraph of section 12 may be helpful here. that:

All the systems studied later have the property

For each destination, either all mail to that destination is sorted by destination on the primary, or all mail to that destination goes into residue on the primary and is then given a secondary sort.

We shall therefore assume this property in (mentally) constructing a hypothetical "minimum-cost" system. Since this is to be a minimum-cost system, we can also suppose (to minimize the cost of residue bins) that:

All residue bins are operating at their maximum capacity (see (5.5)) of 1,200 letters/hr.

$(9.2)$

Once this assumption is made, it follows (in order to minimize loading complex costs) that:

A letter which is to go into residue will be dropped into a residue bin before passing another loading complex.

The method of estimating costs used in section 8 depended on counting the numbers of loading complexes, bins, and sweepers, and adding up the resultant costs. The approach used below is fundamentally different, in that it involves following every letter through the system and assigning an appropriate cost at each stage of its progress. We first consider a letter which enters the system through some loading complex $\mathscr{L}_{1}$ and then (without being dropped) passes a succeeding loading complex $\mathscr{L}_{2}$. This letter prevents $\mathscr{L}_{2}$ from working at its maximum capacity of 36,000 letters/hr; some other letter, which could otherwise have entered the system through $\mathscr{L}_{2}$, will now have to enter the system through some "extra" loading complex (in addition to the minimal 28 complexes known to be required by $(8.3)$ ). If we suppose (to minimize the number of extra complexes and thus the total cost of all extra complexes) that all extra complexes are oper ating at capacity, then our original letter, by passing $\mathscr{L}_{2}$ has created a requirement of " $1 /(36,000)$ of an extra loading complex." Since (by 7.4) each extra loading complex adds $\$ 12,700$ to the system, we are led to the following rule for use in estimating the cost of our hypothetical system:

Assign a cost of $12,700 / 36,000 \approx .353$ whenever a letter passes a loading complex.

Suppose now that it is possible to divide each extra loading complex into 36,000 parts, each able to handle 1 letter $/ \mathrm{hr}$, and that it is possible to add enough of these parts to each of the minimal 28 "nonextra" loading complexes required for the primary sort to ensure that these complexes operate at 
their full 36,000 letters/hr capacity. Such a policy would tend to decrease bin costs and, indirectly, sweeper costs (since bins which previously received fewer than 60 letters/hr might now receive 60 or more; see (5.3)). We therefore assume, for our hypothetical minimum-cost system, that this policy has been adopted, so that the primary sort involves 28 loading complexes (each possibly augmented by parts of extra loading complexes) all working at their maximum capacity. If we also make the "minimumcost" assumption that all loading complexes used for the secondary sort are operating at maximum capacity, then we have a situation in which:

All loading complexes operate at their maximum rate of 36,000 letters $/ \mathrm{hr}$.

$(9.5)$

Thus any letter entering the primary or secondary sort uses $1 /(36,000)$ of the services of the loading complex through which it enters, so that we have the following rule analogous to (9.4):

Assign a cost of .353 whenever a letter enters the primary or secondary sort.

The essential assumptions made so far are (9.1), $(9.2),(9.3)$, and (9.5). Before proceeding further, we point out that two of the (physically realizable) types of sorting configurations to be analyzed later actually $d o$ satisfy these assumptions. The simplex scheme (treated in sec. 10) satifies them exactly, obeying vacuously the conditions referring to residue; the optimal residue scheme found in section 12 satisfies them very nearly. 'Thus these schemes can be treated by the method developed below. ${ }^{7}$ There is no need (see sec. 10) for such a detailed treatment of the simplex scheme. We shall treat the residue scheme, however; in fact, since the methods of this section are less obviously correct than is the "count the sweepers, bins, and loading complexes" approach, we will later analyze the residue scheme using each approach separately and verify that the same answer is obtained in both cases.

Returning to the analysis, we define

$$
\begin{gathered}
f_{i}=\text { fraction of the mail which goes to the } i \text { th } \\
\text { destination }
\end{gathered}
$$

and consider whether or not letters to the $i$ th destination should go into residue on the primary sort. For this purpose it is convenient to define

$$
V_{i}= \begin{cases}1,000,000 f_{i} & \text { if } 1,000,000 f_{i} \leq 60, \\ 60 & \text { otherwise }\end{cases}
$$

and to note that (by (6.1))

$$
1,000,000 f_{i}=\begin{gathered}
\text { number of letters } / \text { hr } \\
\text { destination }
\end{gathered} \text { to the } i \text { th }
$$

In addition, from (7.4) and (5.2) we are led to the rule:

${ }_{7}$ Actual schemes involving multiple input fail to obey (9.5); in treating these schemes by the methods developed below, one must take into account the actual input rates of the loading complexes.
Assign a (personnel) cost of $14,670 / 180=81.50$

for each stack to be swept.

If letters to the $i$ th destination go into residue, then the total cost to be assigned to each such letter as it travels through the sorting system can be calculated in the following way. The letter enters the primary and secondary sorts, so $(9.6)$ yields a loading complex cost of $2 \times(.353)$. By (9.3), there is no contribution from $(9.4)$. By $(9.2)$ and (7.4), the letter should be "charged" $14 / 1,200$ for its use of a residue bin. By (5.5) and (7.4), assuming $10^{6} f_{i} \leq 1,200$, it should be charged $14 / 10^{6} f_{i}$ for its use of a bin in the secondary sort. By (5.1) and (9.10) it should be charged $81.50 / 60$ for the sweep of its residue bin, while by (5.1), (5.3) and (9.10) it should be charged $81.50 / V_{i}$ for the sweep of its bin in the secondary sort. Adding together these partial costs and defining

$\begin{aligned} C\left(f_{i}\right)= & \text { cost to be assigned to a letter to the } i \text { th } \\ & \text { destination, if such letters go in to residue, }\end{aligned}$

we have

$$
\begin{gathered}
C\left(f_{i}\right)=\left(14 / 10^{6} f_{i}\right)+\left(81.50 / V_{i}\right)+2.076 \\
\text { (if } \left.10^{6} f_{i} \leq 1,200\right) .
\end{gathered}
$$

Next we want to calculate the analogous cost if letters to the $i$ th destination do not go into residue for a secondary sort. The primary sort of our hypothetical system now consists of 28 (possibly augmented) loading complexes in series, each followed by a row of bins (some of which may be residue bins); this series arrangement involves no loss in generality, since any other arrangement can be obtained as a special case. ${ }^{8}$ We note first that

bins for the $i$ th destination are spaced uniformly through the primary (except possibly at the end).

To see why this is so, suppose for example that it is found that the first bins for the $i$ th destination should be placed after the third loading complex. Then in view of (9.5), the situation regarding this destination is the same beginning with the fourth loading complex as it was beginning with the first complex, and for the same reasons as before we would find that the next bins for the destination should be placed as far after the fourth complex as the first bins were placed after the first complex. Thus we can define

$$
\begin{aligned}
n_{i}= & \text { number of loading complexes between suc- } \\
& \text { cessive appearances in the primary of bins } \\
& \text { for the } i \text { th destination. }
\end{aligned}
$$

In view of (9.5), a letter to the $i$ th destination is equally likely to pass $0,1,2, \ldots, n_{i}-1$ complexes, each with a probability $1 / n_{i}$, so that the average cost contribution due to (9.4) is

${ }^{8}$ For example, to obtain as a special case (so far as cost factors are concerned) a multiple input system involving two series of 14 loading complexes each, we simply assume in our hypothetical system that all mail is dropped between the 14 th and 15 th complexes. 
$(.353) \times\left(0+1+2+\ldots+\left(n_{i}-1\right)\right) / n_{\imath}=0.1765\left(n_{i}-1\right)$,

where the formula for the sum of an arithmetic progression has been used. There is a contribution of .353 due to (9.6). The system contains $28 / n_{i}$ sets of bins for the $i$ th destination, each set (disregarding fractional effects) receiving

$$
1,000,000 f_{i} /\left(28 / n_{i}\right) \text { letters } / \text { hr; }
$$

if we define

$$
V_{i}^{\prime}=\left\{\begin{array}{ll}
1,000,000 f_{i} /\left(28 / n_{i}\right) & \text { if this is } \leq 1,200, \\
1,200 & \text { otherwise, }
\end{array}(9.15)\right.
$$

the let ter should be charged (again ignoring fractional effects ) $14 / V_{i}^{\prime}$ for its use of a bin. Similarly, if we define

$$
V_{i}^{\prime \prime}= \begin{cases}1,000,000 f_{i} /\left(28 / n_{i}\right) & \text { if this is } \leq 60, \\ 60 & \text { otherwise, }\end{cases}
$$

then the letter should be charged (see (9.11)) $81.50 / V_{i}^{\prime \prime}$ for the sweep of its bin. Adding together these partial costs and defining

$$
\begin{aligned}
C\left(f_{i}, n_{i}\right)= & \text { cost to be assigned to a letter to the } \\
& i \text { th destination, if such letters are }
\end{aligned}
$$
sorted directly on the primary,

we have

$$
\begin{aligned}
C\left(f_{i}, n_{i}\right)=0.1765 n_{i}+\left(14 / V_{i}^{\prime}\right) & +\left(81.50 / V_{i}^{\prime \prime}\right) \\
& +0.1765 .
\end{aligned}
$$

We have now developed the formulas needed to investigate whether or not mail to a given destination should be put into residue. It is convenient to divide the destinations into three classes:

Class 1.

Class 2.

Class 3.

$$
\begin{aligned}
& 1,000,000 f_{i} \leq 60, \\
& 60<1,000,000 f_{i} \leq 1,200, \\
& 1,200<1,000,000 f_{i} .
\end{aligned}
$$

In each class, the "into-residue" cost $C\left(f_{i}\right)$ should be compared with the "not-into-residue" cost $C\left(f_{i}, n_{i}\right)$ evaluated at that value of the system design parameter $n_{i}$ which minimizes it.

Analysis of class 1 . Since the first alternative of (9.8) holds, (9.12) yields

$$
C\left(f_{i}\right)=2.076+\left(0.955 \times 10^{-4}\right) / f_{\imath} .
$$

Since the first alternatives of (9.15) and (9.16) apply, (9.18) yields

$$
C\left(f_{i}, n_{i}\right)=0.1765 n_{i}+\left(2.674 \times 10^{-3}\right) /\left(n_{i} f_{i}\right)+0.1765,
$$

so that $\partial C\left(f_{i}, n_{i}\right) / \partial n_{i}=0.1765-2.674 \times 10^{-3} /\left(n_{i}^{2} f_{i}\right)$, from which it follows that $C\left(f_{i}, n_{i}\right)$ is a decreasing function of $n_{i}$ for

$$
n_{i}^{2} \leq 1.515 \times 10^{-2} / f_{i}
$$

i.e., for

$n_{\imath} \leq 0.1231 / \sqrt{f_{i}}$,

and is increasing for higher values of $n_{i}$. Since $n_{i} \leq 28$, the minimizing value of $n_{i}$ is

$$
\begin{cases}0.1231 / \sqrt{f_{i}} & \text { if this is } \leq 28, \\ 28 & \text { otherwise, }\end{cases}
$$

i.e., is

$$
\begin{cases}0.1231 / \sqrt{f_{i}} & \text { if } f_{i} \geq 4.439 \times 10^{-3} \\ 28 & \text { otherwise. }\end{cases}
$$

By (9.20) and (9.19)

$$
C\left(f_{i}, 28\right)=5.1185+\left(0.955 \times 10^{-4}\right) / f_{i}>C\left(f_{i}\right),
$$

so that if the second alternative of (9.21) holds then mail to the $i$ th destination should go into residue. If the first alternative holds in (9.21) then

$C\left(f_{i}, n_{i}\right)_{\mathrm{min}}=C\left(f_{i}, \quad 0.1231 / \sqrt{f_{i}}\right)=0.1765+\left(.04345 / \sqrt{f_{i}}\right)$, and (from (9.21) and the fact $1,000,000 f_{i} \leq 60$ )

$$
\begin{gathered}
4.439 \times 10^{-3} \leq \sqrt{\overline{f_{i}}} \leq 7.746 \times 10^{-3}, \\
129.1 \leq x=\left(1 / \sqrt{f_{i}}\right) \leq 225.3
\end{gathered}
$$

From (9.19) and our expression for $C\left(f_{i}, n_{i}\right)_{\min }$, we then have

$$
C\left(f_{i}, n_{i}\right)_{\min }-C\left(f_{i}\right)=0.04345 x-0.955 \times 10^{-4} x^{2}-1.8955 ;
$$

this function of $x$ is increasing (i.e., its derivative is positive) in the range (9.22) and is $>0$ at $x=129.1$; thus it is positive throughout the range (9.22), which shows that even if the first alternative of $(9.21)$ holds, mail to the $i$ th destination should go into residue. Thus in our minimum-cost system, all mail to class 1 destinations should go into residue.

For our particular mail distribution (see app. A) the class 1 destinations are destinations 901 to 1,600, and these 700 destinations receive 18,080 letters $/ \mathrm{hr}$. These letters lead via (9.6) to a cost of

$$
2 \times(.353) \times 18,080
$$

associated with loading complexes, of

$$
14 \times(18,080 / 1,200)+14 \times 700
$$

associated with residue bins and secondary bins, and of

$$
81.50 \times(18,080 / 60)+81.50 \times 700
$$

associated with sweeps of residue bins and secondary bins. Adding these, we find that in our minimum- 
cost system class 1 destinations involve a total cost of approximately

$$
\$ 104,400 \text {. }
$$

Analysis of class 2. Since the second alternative of (9.8) holds, (9.12) yields

$$
C\left(f_{i}\right)=\left(14 / 10^{5} f_{i}\right)+3.434 .
$$

The first alternative of (9.15) holds, but either alternative of (9.16) may apply, so that (9.18) yields

$$
\begin{aligned}
& C\left(f_{i}, n_{i}\right)=0.1765 n_{i}+\left(2.674 \times 10^{-3}\right) /\left(n_{i} f_{i}\right)+0.1765 \\
& \text { (if } n_{i} \leq 1680 / 10^{6} f_{i} \text { ), } \\
& C\left(f_{i}, n_{i}\right)=0.1765 n_{i}+\left(0.392 \times 10^{-3}\right) /\left(n_{i} f_{i}\right)+1.5348 \\
& \text { (if } n_{i}>1,680 / 10^{6} f_{i} \text { ). }
\end{aligned}
$$

As in the analysis of class 1 , the function given in $(9.25)$ is a decreasing function of $n_{i}$ for

$$
n_{i} \leq 0.1231 / \sqrt{f_{i}}
$$

and is increasing for higher values of $n_{i}$. On the other hand, the function given by $(9.26)$ is a decreasing function of $n_{i}$ for

$$
n_{i} \leq 0.04713 / \sqrt{f_{i}}
$$

and is increasing for higher values of $n_{i}$.

For further analysis it is convenient to divide class 2 into subclasses, depending on the relative positions of $n_{i}=0.1231 / \sqrt{f_{i}}$ (where the function given by $(9.25)$ has its minimum), $n_{i}=0.04713 / \sqrt{f_{i}}$ (where the function given by $(9.26)$ has its minimum), and $n_{i}=1,680 / 10^{6} f_{i}$ (where the formula for $C\left(f_{i}, n_{i}\right)$ changes from $(9.25)$ to $\left.(9.26)\right)$. This subdivision leads to

Class $2 a . \quad 60<1,000,000 f_{i} \leq 186.3$,

Class 2b. $\quad 186.3<1,000,000 f_{i} \leq 1,200$,

corresponding respectively (subject to $60<10^{6} f_{i} \leq$ $1,200)$ to

$$
\begin{aligned}
& 0.04713 / \sqrt{f_{i}}<0.1231 / \sqrt{f_{i}} \leq 1,680 / 10^{6} f_{i}, \\
& 0.04713 / \sqrt{f_{i}} \leq 1,680 / 10^{6} f_{i}<0.1231 / \sqrt{f_{i}} .
\end{aligned}
$$

Analysis of class 2a. Here $C\left(f_{i}, n_{i}\right)_{\mathrm{m} \text { in }}$ must arise either by setting $n_{i}=0.1231 / \sqrt{f_{i}}$ in $(9.25)$, yielding

$$
0.1765+\left(0.04345 / \sqrt{f_{i}}\right),
$$

or by setting $n_{i}=1,680 / 10^{6} f_{i}$ in (9.26), yielding

$$
1.7681+\left(296.5 / 10^{6} f_{i}\right) .
$$

It turns out that the first form is the smaller, so that, combining the first form with (9.24), we have
$C\left(f_{i}, n_{i}\right)_{\mathrm{m} \text { in }}-C\left(f_{i}\right)=\left(0.04345 / \sqrt{f_{i}}\right)-\left(14 / 10^{6} f_{i}\right)-3.258$.

This function of $f_{i}$ is positive for

$$
60<1,000,000 f_{i}<168.2,
$$

which shows that mail to the $i$ th destination should go into residue if $(9.31)$ holds.

For our particular mail distribution, destinations 526 to 900 are the ones obeying (9.31); these 375 destinations receive 40,250 letters/hr, leading to a cost of

$$
2 \times(.353) \times(40,250)
$$

associated with loading complexes, of

$$
12 \times(40,250 / 1,200)+14 \times 375
$$

associated with residue bins and secondary bins, and of

$$
81.50 \times(40,250 / 60)+81.50 \times(40,250 / 60)
$$

associated with sweeps of residue bins and secondary bins. Adding these, we find that in our hypothetical minimum-cost system the mail to destinations obeying (9.31) has a total associated cost of approximately

$$
\$ 143,500 \text {. }
$$

Before proceeding further, we will write out explicitly a principle which will be helpful in much of the following work. The correctness of the principle could be proved analytically in each situation in which we invoke it, but such proofs would be lengthy and repetitious, and so we content ourselves here with stating the principle (which is intuitively evident anyhow). Informally, the principle simply recognizes the fact that bins for destinations receiving a good deal of mail should appear rather frequently in the primary of our minimum-cost system (i.e., $n_{i}$ will be small), bins for destinations receiving less mail should appear less frequently, (i.e., $n_{i}$ will be larger), and there is a "threshhold $t$ " such that the destinations whose mail is put into residue are precisely those which receive fewer than $t$ letters/hr. Formally:

Suppose it is best in our minimum-cost system that mail to the $i$ th destination not go into residue, and suppose that $\left(n_{i}\right)_{\text {opt }}$ is the best value of $n_{i}$. Then for any other destination with $f_{j} \geq f_{i}$, it is best that mail to this destination also not go into residue, and furthermore $\left(n_{j}\right)_{\mathrm{opt}} \leq\left(n_{i}\right)_{\mathrm{opt}}$.

Returning to the analysis, we now consider the class 2 a destinations not obeying (9.31); i.e., those for which

$$
168.2 \leq 1,000,000 f_{i} \leq 186.3 .
$$

For these destinations, the function of $f_{i}$ given in (9.30) is negative, but we cannot automatically con- 
clude that mail to these destinations should not go into residue. The difficulty is that we treated $n_{i}$ as a continuous variable in finding $C\left(f_{i}, n_{i}\right)_{\mathrm{min}}$, whereas in fact $n_{i}$ must be a positive integer. We therefore define (for fixed $f_{i}$ )

$C\left(f_{i}, n_{i}\right)_{\min }^{(\text {int) }}=$ minimum of $C\left(f_{i}, n_{i}\right)$ for all positive integer values of $n_{i}$;

the value of $n_{i}$ yielding $C\left(f_{i}, n_{i}\right)_{\text {min }}^{(\text {int) }}$ will be one of the integers between which the $n_{i}$ yielding $C\left(f_{i}, n_{i}\right)_{\mathrm{min}}$ lies.

We begin at the "top" of (9.34), with

$$
1,000,000 f_{i}=186.3 \text {. }
$$

The value of $n_{i}$ yielding $C\left(f_{i}, n_{i}\right)_{\min }$ is

$$
n_{i}=0.1231 / \sqrt{f_{i}}=9^{+},
$$

so that $\left(n_{i}\right)_{\text {opt }}$ is either 9 or 10 . From (9.24) we have

$$
C\left(f_{i}\right)=C\left(186.3 \times 10^{-6}\right) \approx 3.51 .
$$

Substituting $n_{i}=9$ and $n_{i}=10$ into the applicable choices of $(9.25)$ and $(9.26)$ (since $1,680 / 10^{6} f_{i}=9^{+}$, $n_{i}=9$ goes into $(9.25)$ and $n_{i}=10$ into $(9.26)$ ), we obtain costs of approximately

$$
3.34 \text { for } n_{i}=9, \quad 3.51 \text { for } n_{i}=10,
$$

so that $\left(n_{i}\right)_{\text {opt }}=9$ and mail to destinations obeying (9.36) should not go into residue in our hypothetical minimum-cost system. We note for future reference that, by (9.33), all destinations in classes $2 \mathrm{~b}$ and 3 should not have their mail sent in to residue, and that throughout these classes $\left(n_{i}\right)_{\text {opt }} \geq 9$.

We now continue "down" through (9.34), considering lower values of $f_{i}$. Throughout (9.34),

$$
n_{i}=0.1231 / \sqrt{f_{i}}=9^{+}
$$

so that $\left(n_{i}\right)_{\text {opt }}$ is either 9 or 10 ; also, throughout (9.34) we have $1,680 / 10^{6} f_{i}=9^{+}$, so that $n_{i}=9$ must be substituted into $(9.25), n_{i}=10$ must be substituted into (9.26), and the results compared with the result of $(9.24)$. Since this yields

$$
\begin{aligned}
& C\left(f_{i}, 9\right)=1.7650+\left(297.1 / 10^{6} f_{i}\right), \\
& C\left(f_{i}, 10\right)=3.2998+\left(39.2 / 10^{6} f_{i}\right),
\end{aligned}
$$

we find (using (9.24) also) that mail should go into residue for

$$
168.2 \leq 1,000,000 f_{i} \leq 169.6
$$

and should not go into residue for

$$
169.6<1,000,000 f_{i} \leq 186.3,
$$

and also that

$$
\left(n_{i}\right)_{\mathrm{opt}}=9 \quad \text { in }(9.38) .
$$

For our particular mail distribution, there are no destinations in the narrow range (9.37). The cost associated with destinations obeying (9.38) will not be found here, since it can more conveniently be combined with the cost found for class $2 b$.

Analysis of class $2 b$. We know that mail to these destinations does not go into residue. Also, we know that at the "bottom" of class 2 b (i.e., $10^{6} f_{i}=$ 186.3) we have $\left(n_{i}\right)_{\mathrm{opt}}=9$, and from $(9.33)$ we can expect that as we search "upward" through class $2 \mathrm{~b}$ (i.e., examine successively larger values of $f_{i}$ ) we will reach a point where $\left(n_{i}\right)_{\text {opt }}$ changes from 9 to 8. Our immediate aim is to find this turnover point.

We find that for $186.3 \leq 10^{6} f_{i} \leq 186.7, C\left(f_{i}, 8\right)$ and $C\left(f_{i}, 9\right)$ are both given by $(9.25)$, so that

$$
\begin{aligned}
& C\left(f_{i}, 9\right)=1.7650+\left(297.1 / 10^{6} f_{i}\right) \\
& C\left(f_{i}, 8\right)=1.5885+\left(334.3 / 10^{6} f_{i}\right)
\end{aligned}
$$

Throughout the range $C\left(f_{i}, 9\right)$ is smaller, so that

$$
\left(n_{i}\right)_{\mathrm{opt}}=9 \quad \text { for } \quad 186.3 \leq 10^{6} f_{i} \leq 186.7 \text {. }
$$

For $186.7<10^{6} f_{i} \leq 210, \quad C\left(f_{i}, 8\right)$ is still given by $(9.25)$ but $C\left(f_{i}, 9\right)$ is now given by $(9.26)$, so that

$$
C\left(f_{i}, 9\right)=3.1233+\left(43.56 / 10^{6} f_{i}\right) .
$$

Comparing (9.40) and (9.41), we find that

$$
\left(n_{i}\right)_{\mathrm{opt}}=9 \quad \text { for } \quad 186.7<10^{6} f_{i} \leq 189.4
$$

but

$$
\left(n_{i}\right)_{\mathrm{opt}}=8
$$

for values of $10^{6} f_{i}$ immediately above 189.4.

We can continue to search upwards through class $2 \mathrm{~b}$, looking next for the turnover from $\left(n_{i}\right)_{\text {opt }}=8$ to $\left(n_{i}\right)_{\text {opt }}=7$, etc. The results of this search are given in table 1 which includes the class 2a destinations obeying (9.38). The quantity $10^{6} f_{i}$ has been rounded.

The first three columns of table 1 are independent of the particular mail distribution. The first, third, fourth, and fifth columns can be used to derive a cost figure in the following way: For each row, the fourth entry shows how many destinations are involved and the first entry enables us to find how many bins to each destination appear in our minimum-cost system. If (9.25) holds, then these bins are each swept once an hour, whereas if (9.26) holds then the number of sweeps involved is the same as that used in deriving (8.7); we can use the fifth entries of the rows involving (9.25) to find the numbers of "extra sweepers" involved (see (8.11)) and then multiply this contribution to $\Delta S$ by 14,670 in accordance with (8.12). Finally, we can use the first and fifth columns, together with (9.4) and (9.6), to assign a loading complex cost to each row. The result ${ }^{9}$ is a total cost of about

${ }^{9}$ Different methods of associating integral numbers of bins with values of $n$, which are not divisors of 28 were tested and found to change (9.42) only negligibly. 
$\$ 401,200$

$(9.42)$

associated with class $2 \mathrm{~b}$ destinations and the class $2 \mathrm{a}$ destinations obeying (9.38).

TABLE 1. Optimizing $n_{i}$ in class $2 b$

\begin{tabular}{|c|c|c|c|c|}
\hline$\left(n_{i}\right)_{\mathrm{opt}}$ & $10^{6} f_{i}$ & Formula & Destinations & Letters/hr \\
\hline 9 & 170 to 187 & (9. 25) & 491 to 525 & 5,950 \\
\hline 9 & 188 to 189 & $(9.26)$ & & \\
\hline 8 & 190 to 209 & $(9.25)$ & 451 to 490 & 7,700 \\
\hline 8 & 210 to 217 & $(9.26)$ & 441 to 450 & 2,100 \\
\hline 7 & 218 to 239 & $(9.25)$ & 421 to 440 & 4,500 \\
\hline 7 & 240 to 254 & $(9.26)$ & 401 to 420 & 4,900 \\
\hline 6 & 255 to 279 & $(9.25)$ & 381 to 400 & 5,300 \\
\hline 6 & 280 to 305 & $(9.26)$ & 351 to 380 & 8,800 \\
\hline 5 & 306 to 335 & $(9.25)$ & 341 to 350 & 3,200 \\
\hline 5 & 336 to 384 & $(9.26)$ & 311 to 340 & 10,800 \\
\hline 4 & 385 to 419 & $(9.25)$ & 300 to 310 & 4,510 \\
\hline 4 & 420 to 516 & $(9.26)$ & 265 to 299 & 16. 120 \\
\hline 3 & 517 to 559 & $(9.25)$ & 253 to 264 & 6,300 \\
\hline 3 & 560 to 786 & $(9.26)$ & 203 to 252 & 32,720 \\
\hline 2 & 787 to 839 & $(9.25)$ & 200 to 202 & 2,420 \\
\hline 2 & 840 to 1,200 & $(9.26)$ & 154 to 199 & 46,400 \\
\hline \multicolumn{4}{|c|}{ Total number of letters $/ \mathrm{hr}$} & 161,720 \\
\hline
\end{tabular}

Analysis of class 3. Here the different possible alternatives in (9.15) and (9.16) lead to three possible formulas for $C\left(f_{i}, n_{i}\right)$ :

$$
\begin{aligned}
& C\left(f_{i}, n_{i}\right)=0.1765 n_{i}+\left(2.674 \times 10^{-3}\right) /\left(n_{i} f_{i}\right)+0.1765 \\
& \text { (if } n_{i} \leq 1,680 / 10^{6} f_{i} \text { ), } \\
& C\left(f_{i}, n_{i}\right)=0.1765 n_{i}+\left(0.392 \times 10^{-3}\right) /\left(n_{i} f_{i}\right)+1.5348 \\
& \text { (if } 1,680 / 10^{6} f_{i}<n_{i} \leq 33,600 / 10^{6} f_{i} \text { ), } \\
& C\left(f_{i}, n_{i}\right)=0.1765 n_{i}+1.546 \\
& \text { (if } n_{i}>33,600 / 10^{6} f_{i} \text { ). }
\end{aligned}
$$

We can work "upwards" through class 3 just as we did in class $2 \mathrm{~b}$, beginning with $\left(n_{i}\right)_{\text {opt }}=2$; the only new complication is that possible transitions to (9.45) must be allowed for. It turns out that the change from $\left(n_{i}\right)_{\mathrm{opt}}=2$ to $\left(n_{i}\right)_{\mathrm{opt}}=1$ occurs for $10^{6} f_{i}=1,614$ (i.e., at destination 119 in our particular mail distribution) and that the cost associated with class 3 destinations is

$$
\$ 1,397,000 \text {. }
$$

Finally, we add up (9.23), (9.32), (9.42), and (9.46) to obtain (after rounding off) a total of

$$
C_{\min }^{*}=\$ 2,046,000
$$

as the approximate cost of our hypothetical minimum-cost system. This is then our theoretical minimum cost, to be used as a yardstick in dealing with proposed sorting configurations. It is significantly larger than (8.8).

The general approach used in this section constitutes the "follow through" method mentioned in section 1 .

\section{Simplex Scheme}

The first sorting scheme we examine is also the simplest. The sorting system consists of a number of essentially independent subsystems, each consist- ing of a single loading complex followed by 1,600 bins, one for each of the 1,600 destinations.

The argument used to derive (8.3) shows that for this system $L=28$, so that

$$
\Delta L=0 ;
$$

thus there are 28 subsystems, so that

$$
\Delta B=(28) \times(1,600)-840 \approx 44,000 .
$$

Assuming the 1,000,000 letters/hr divide equally (on the average) among the 28 subsystems, we find that a bin corresponding to one of the 114 most frequent destinations will receive 60 or more letters/hr, whereas a bin corresponding to one of the $(1,600-114)=1486$ least frequent will receive fewer than 60 . There are

$$
1,486 \times 28 \approx 41,600
$$

bins in the system which correspond to these last 1,486 destinations, and by (5.3) these bins give rise to $41,600 \mathrm{stacks} / \mathrm{hr}$. These last 1,486 destinations receive only 27.6 percent of the total mail $(276,000$ letters/hr) and so, if all stacks consisted of 60 letters (the basis on which (8.5) was derived), they would give rise to only

$$
276,000 / 60=4,600 \text { stacks } / \mathrm{hr} .
$$

Thus we have $41,600-4,600=37,000$ extra stacks $/ \mathrm{hr}$, leading via (5.2) to

$$
37,000 / 180 \approx 205
$$

additional sweepers, of which two were taken into account in passing from (8.5) to (8.7). So

$$
\Delta S=203
$$

and by (8.12)

$$
\Delta C=(14) \times(44,000)+(14,670) \times(203) \approx 3,594,000,
$$

so that, by (8.8) and (8.13),

$$
C=C_{\min }+(\Delta C)=5,355,000,
$$

and by (9.47),

$$
\left(C-C_{\min }^{*}\right) / C_{\min }^{*} \approx 162 \% .
$$

Thus on a cost basis the simplex scheme should definitely be rejected. The excessive cost comes primarily from personnel, i.e., from the second summand in (10.4).

\section{Christmas Tree Scheme}

This might also be called the "square root" scheme. In our case the square root of the number 
of destinations is $\sqrt{1,600}=40$. The system consists of a number of subsystems, each containing a single loading complex and 40 bins. ${ }^{10}$ The 1,600 destinations are divided into 40 groups of 40 destinations each; the primary sorting of mail is done by group, and the mail to each group is then given a secondary sort by individual destinations.

The key to the analysis is the fact that each piece of mail is dealt with twice. By applying (8.3), (8.4), and (8.5) to both the primary and the secondary sorts, and adding the results, we have

so that

$$
L \geq 56, \quad B \geq 1,680, \quad S \geq 186,
$$

$$
\Delta L \geq 28, \quad \Delta B \geq 840, \quad \Delta S \geq 91
$$

and by (8.12)

$$
\Delta C \geq 1,702,000,
$$

so that by (8.8) and (8.13)

and

$$
C \geq 3,463,000
$$

$$
\left(C-C_{\min }^{*}\right) / C_{\min }^{*}>69 \% \text {. }
$$

On this basis we can reject the Christmas Tree Scheme. Again personnel costs are the major factor.

\section{Residue Scheme}

In this system the primary sort consists of sorting letters directly to those destinations which receive a relatively large fraction of the mail, while dropping all letters to the less "frequent" destinations into a relatively small number of residue bins, which are then given a secondary sort by destination. The purpose of this maneuver is to avoid having a large number of sweeps (corresponding to infrequent destinations) resulting in small stacks; such sweeps lead to excessive personnel costs.

The system is determined by stating definitely which destinations are to be considered "infrequent" (so that mail to them goes into residue) and which are to be considered "frequent." We therefore define a system design parameter

$t=$ threshold; the $i$ th destination is frequent if $10^{6} f_{i} \geq t, \quad$ infrequent if $10^{6} f_{i}<t$;

the value of $t$ is to be chosen so as to minimize the cost of the system. As before,

$f_{i}=$ fraction of mail which goes to the $i$ th destination.

$(12.2)$

We shall determine the optimal value of $t$ by two methods (in order to check their agreement). First we apply the "follow through" approach of section 9 . The residue scheme certainly satisfies (9.1) and (9.3); if well-designed, it will very nearly satisfy

10 The cost estimate would be even higher if we took into account the indivisibility of the 30-bin modules.
(9.2) and (9.5) as well. We will therefore proceed as in section 9 . The number of letters/hr to the $i$ th destination in each of the 28 primary subsystems is

$$
1,000,000 f_{i} / 28
$$

and if this quantity is $>60$ then by (5.4) there would be no reason to put mail to the $i$ th destination into residue. Thus the only destinations about which there is any question are those for which

$$
\left(1,000,000 f_{i} / 28\right) \leq 60 .
$$

We can split these destina tions into classes, according as

$$
\begin{array}{cc}
\text { or } & 1,000,000 f_{i} \leq 60, \\
\text { or } & 60<1,000,000 f_{i} \leq 1,200, \\
& 1,200<1,000,000 f_{i} \leq 1,680 .
\end{array}
$$

For destinations obeying (12.3a), (9.12) yields

$$
C\left(f_{i}\right)=2.076+\left(95.50 / 10^{6} f_{i}\right),
$$

while (9.18), since $n_{i}=1$ in the residue scheme, yields

$$
C\left(f_{i}, 1\right)=0.353+\left(2674 / 10^{6} f_{i}\right) .
$$

Similarly, for destinations obeying $(12.3 \mathrm{~b})$ we have

$$
\begin{gathered}
C\left(f_{i}\right)=3.434+\left(14 / 10^{6} f_{i}\right) \\
C\left(f_{i}, 1\right)=0.353+\left(2,674 / 10^{6} f_{i}\right) .
\end{gathered}
$$

For destinations obeying (12.3c), it is not clear in view of $(5.5)$ what a letter going into residue should be "charged" for the sweep of its secondary bin; it should be at least 14/1,200, however, so that (see $(9.25)$ ) we have

and

$$
C\left(f_{i}\right)<(14 / 1,200)+3.434=3.451,
$$

$$
C\left(f_{i}, 1\right)=1.7113+\left(392 / 10^{6} f_{i}\right) .
$$

We find that $C\left(f_{i}\right)-C\left(f_{i}, 1\right)$ is negative (i.e., it is less expensive to put mail to the $i$ th destination into residue) for $10^{6} f_{i} \leq 863$ and is positive for $10^{6} f_{i} \geq$ 864. Thus the optimal $t$ is

$$
t_{\mathrm{opt}}=864 \text {, }
$$

which for our particular mail distribution corresponds to destination 196.

Next we apply the "add up" method based on (8.12). First we define two relevant quantities, depending on $t$, by

$N_{t}=$ number of frequent destinations,

$V_{t}=$ number of letters/hr to frequent destinations,

so that (since our situation involves in all $1,000,000$ 
letters/hr and 1,600 destinations).

$1,600-N_{t}=$ the number of infrequent destinations, $1,000,000-V_{t}=$ number of letters $/ \mathrm{hr}$ to infrequent destinations.

The number $\Delta L$ of extra loader complexes needed for the secondary sort is given (using (8.2)) by

$$
\Delta L \approx\left(1,000,000-V_{t}\right) / 36,000 ;
$$

more precisely, $\Delta L$ is the integer next above the right side of (12.7). The infrequent destinations are divided into $\Delta L$ groups whose total expected hourly mail volumes are approximately equal.

The sorting system contains $28+(\Delta L)$ sub. systems, each consisting of a loading complex followed by a number of bins. Each of the 28 subsystems needed for the primary sort contains $N_{t}$ bins for the frequent destinations and one residue bin $^{11}$ for each of the $\Delta L$ groups of infrequent destinations. Each of the $\Delta L$ subsystems used for the secondary sort, sorts the entire primary residue of some one of the groups; thus these $\Delta L$ subsystems together contain one bin ${ }^{11}$ for each infrequent destination, and thus contain $1,600-N_{t}$ bins in all. Therefore

$$
\begin{aligned}
B & =28\left(N_{t}+(\Delta L)\right)+\left(1,600-N_{t}\right) \\
& =28(\Delta L)+27 N_{t}+1,600 \\
\Delta B & =B-840=28(\Delta L)+27 N_{t}+760 .
\end{aligned}
$$

Having found $\Delta L$ and $\Delta B$, we must find $\Delta S$. The frequent destinations receiving 60 or more letters $/ \mathrm{hr}$ on each of the 28 primary subsystems, require no more sweeps than was assumed in calculating the minimum cost. The same holds for secondary sweeps of mail to the infrequent destinations. The extra sweeps therefore arise $(a)$ from sweeping the residue bins and $(b)$ from the primary sweeps of frequent destinations receiving fewer than 60 letters/hr on each of the 28 primary-sorting subsystems; the frequent destinations described in $(b)$ are those receiving fewer than $(28) \times(60)=1,680$ letters $/ \mathrm{hr}$ in all, and thus those whose mail frequencies are less than 0.168 percent. In our actual mail distribution, 114 destinations have frequencies of 0.168 percent or more, and these destinations account for 72.40 percent of the total mail. Thus

$N_{t}-114=$ number of destinations of the type (b), $(12.9)$

$V_{t}-724,000=$ hourly volume of mail to these destinations.

The destinations described in $(b)$ thus lead to approximately

$$
28\left(N_{t}-114\right) / 180 \text { sweepers }
$$

\footnotetext{
${ }^{11}$ Some extra bins may be required in order to satisfy (5.5), but their cost is negligible.
}

of which $\left(\left(V_{t}-724,000\right) / 60\right) / 180$ were accounted for in calculating (8.8). The number of extra sweepers due to source $(b)$ is therefore approximately

$$
\left(1,680 N_{t}-V_{t}+532,500\right) /(60)(180) .
$$

The residue bins together receive $1,000,000-V_{t}$ letters $/ \mathrm{hr}$ and each receives more than 60 letters $/ \mathrm{hr}$, so that the number of extra sweepers due to source (a) is approximately

$$
\left(1,000,000-V_{t}\right) /(60) \times(180) ;
$$

adding the last two expressions gives

$$
\Delta S \approx\left(1,532,500-2 V_{t}+1,680 N_{t}\right) /(60)(180) .
$$

We now substitute (12.8) and (12.11) into (8.12) obtaining

$$
\Delta C \approx 13,100(\Delta L)+2,660 N_{t}-2.717 V_{t}+2,092,500 .
$$

The error involved in using (12.7) is at most unity, leading to an error of at most 13,100 in (12.12) and thus to an error of less than 1 percent in $\left(C-C_{\min }^{*}\right) / C_{\min }^{*}$. We therefore substitute (12.7) into (12.12) getting

$$
\Delta C \approx 2,660 \quad N_{t}-3.081 \quad V_{t}+2,456,400 .
$$

To minimize $\Delta C$ quickly (we omit the rigorous justification of the following method), equate the differential of the right side of (12.13) to zero:

$$
2,660\left(d N_{t}\right)-3.081\left(d V_{t}\right)=O .
$$

Since $N_{t}$ increases in steps of size 1 (each step involving shifting the status of one destination from "infrequent" to "frequent"), we set $d N_{t}=1$ and obtain

$$
d V_{t}=(2,660) / 3.081 \approx 863 \text { letters } / \text { hr } .
$$

Since the increment in $V_{t}$ due to one extra "frequent" destination is simply the expected hourly volume of mail to that one destination, (12.11) tells us that for the approximate minimization of $\Delta C$, the last of the "frequent" destinations should obey $10^{6} f_{i}=863$, so that (see (12.1))

$$
t_{\mathrm{opt}}=863 \text {, }
$$

in near-perfect agreement with the result (12.6) obtained by the "follow through" method. For our particular mail distribution this "cutoff" occurs between destinations 196 and 197, and after obtaining the value of $V_{t}$ corresponding to $N_{t}=196$ from appendix A and substituting into (12.14) we find (rounding) that

$$
(\Delta C)_{\min }=440,000
$$




$$
\left(C-C_{\min }^{*}\right) / C_{\min }^{*} \approx 8 \% \text {. }
$$

Using (12.11) we find that the cost of personnel is again the main cost factor.

The optimum given by (12.15) (i.e., given by a "cutoff" between destinations 196 and 197) is not a very sharp one; the cutoff can occur as low as about destination 165 or as high as destination 225 without raising $\left(C-\mathrm{C}_{\min }^{*}\right) / C_{\min }^{*}$ to more than 0.5 percent above its minimum.

\section{Multiple-Input Schemes}

In these schemes, the sorting system consists of a number of identical subsystems, each receiving its input from a number of loading complexes arranged in series. More precisely, each subsystem consists of a first loading complex followed by a first row of bins for some (but not all) destinations, then a second loading complex ${ }^{12}$ followed by a second row of bins for some destinations (not necessarily the same ones as in the first row), . . ., and finally a last loading complex followed by a last row of bins for all 1,600 destinations.

Consider some particular sorting system of this type; call it system 1, and let

$$
M=\text { number of subsystems of system } 1 \text {. }
$$

Let system 2 be obtained from system 1 by replacing, with residue bins, the last 700 bins of the last row of each subsystem (these bins correspond to destinations 900 to 1600 , which for our mail distribution are the ones receiving fewer than 60 letters $/$ hr). The residue then requires a secondary sort. Let

$$
\begin{aligned}
R= & \text { number of residue bins/subsystem for } \\
& \text { system } 2 .
\end{aligned}
$$

We will compare the costs of system 1 and system 2 ; let $L_{1}, B_{1}, S_{1}, C_{1}, L_{2}, B_{2}, S_{2}, C_{2}$ denote the respective values of $L, B, S, C$, for the two systems.

Destinations 900 to 1,600 receive about 1.8 percent of the mail, or 18,000 letters/hr; this is less than the 36,000 letters/hr capacity of a loading complex; and so only one complex is needed for the secondary sort:

$$
L_{2}-L_{1}=1
$$

The $700 M$ bins used for destinations 900 to 1,600 in the last rows of the subsystems of system 1 are replaced in system 2 by $R M$ residue bins plus 700 bins for the secondary sort, and so

12 Some of the letters inserted by the first loading complex (namely, letters to those destinations for which bins were not provided in the first row of bins) will still be on the conveyor as it passes the second loading complex. Thus the second loading complex of each subsvstem will not operate at its full rate ( 36,000 letters $/ \mathrm{hr}$ ) and the same holds for the third, fourth, etc. loading complexes of each subsystem.
The above-mentioned $700 M$ bins for system 1 each received fewer than 60 letters/hr, and so together required $700 \mathrm{M}$ sweeps/hr. For system 2, the 700 secondary bins together require 700 sweeps $/ \mathrm{hr}$. As for the $R M$ residue bins, $R$ is chosen as small as possible, so that each residue bin (except possibly for the last one on each subsystem) receives at least 60 letters/hr; the residue bins receive 18,000 letters/hr and thus require approximately

$$
18,000 / 60 \approx 300 \text { sweeps } / \mathrm{hr} \text {. }
$$

Thus we have, using (5.2),

$$
S_{1}-S_{2} \approx(700 M-(300+700)) / 180 \text {. }
$$

The analog of (8.12) which applies to our situation is

$C_{1}-C_{2}=12,700\left(L_{1}-L_{2}\right)+14\left(B_{1}-B_{2}\right)+14,670\left(S_{1}-S_{2}\right)$,

and so, by (13.3), (13.4), and (13.5) we have

$$
C_{1}-C_{2} \approx 67,000 M-14 R M-104,000 .
$$

Since $R$ is chosen as small as (5.5) permits, we have

$$
R M \approx 19,400 / 1,200 \approx 16,
$$

and so (13.7) can be rewritten as

$$
C_{1}-C_{2} \approx 67,000 M-104,000 \text {. }
$$

One rather sweeping conclusion which can be drawn from (13.8) is that no multiple input scheme with more than one subsystem (i.e., with $M>1$ ) can be optimal. To prove this, take the system in question as the "system 1" of the above discussion. Since $M>1,(13.8)$ shows that $C_{1}-C_{2}>0$ and thus that $C_{2}<C_{1}$; since system 2 costs less than system 1 , the latter cannot be optimal. We could use this argument to eliminate multiple input schemes with $M>1$ from further discussion, if we were going later to examine the "systems 2." Unfortunately, the analysis of such systems, which combine elements of the multiple input and residue schemes, appears too complicated to be attempted here (the difficulties of even relatively simple systems of this type will become apparent in sec. 14 and app. C). We can, however, use (13.8) to eliminate all multiple input systems (i.e., all "systems 1") with $M \geq 7$. To do this, we note first that $C_{2}>C_{\min }$, so that

$$
\Delta C_{1}=C_{1}-C_{\min }>C_{1}-C_{2} ;
$$

together with (13.8) and $M \geq 7$, this implies that

$$
\Delta C_{1}>C_{1}-C_{2} \approx 67,000 M-104,000 \geq 365,000,
$$

so that system 1 is at best negligibly less costly than (actually more costly; see footnote 13, p. 96) the 
system to be found in section 14, for which (see $(14.24))$

$$
C \approx 366,000
$$

Now we want to examine multiple-input schemes with $M \leq 6$ (i.e., with six or fewer subsystems). It is convenient to define

$$
\begin{aligned}
N_{M}= & \text { number of destinations receiving fewer } \\
& \text { than } 60 \mathrm{M} \text { letters } / \mathrm{hr}, \\
W_{M}= & \text { number of sweeps associated with these } \\
& \text { destinations in calculating } C_{\min }^{*} .
\end{aligned}
$$

Each such destination receives fewer than 60 letters/ $\mathrm{hr}$ (and thus requires at least one sweep/hr) for each subsystem, so that together these destinations require at least $M N_{M}$ sweeps/hr for the entire system. Thus

$$
\Delta S \geq\left(M N_{M}-W_{M}\right) / 180 .
$$

For $M=6$ we find $M N_{M} \approx 7,620, \quad W_{M} \approx 2,140$, so that, by (8.12),

$$
\begin{array}{r}
\Delta C \geq 14,670 \times 5,480 / 180 \approx 447,000 \\
\text { (for } M=6) .
\end{array}
$$

By comparison with (13.9), systems with $M=6$ are eliminated. ${ }^{13}$ For $M=5$ we find $M N_{M} \approx 6,150$, $W_{M} \approx 1,925$, as well as

$$
\Delta B=B-840 \geq 5 \times 1,600-840=7,160 ;
$$

thus by (8.12),

$$
\begin{array}{r}
\Delta C \geq 14,670 \times 4,225 / 180+14 \times 7,160 \approx 445,000 \\
\text { (for } M=5) . \quad(13 .
\end{array}
$$

By comparison with (13.9), systems with $M=5$ are eliminated.

These relatively simple arguments are not ade. quate to deal with the schemes with $M \leq 4$. Consider first the situation $M=4$. Here $M \bar{N}_{M} \approx 4,720$, $W_{M} \approx 1,710$ so by (13.12)

$$
\Delta S \geq 3,010 / 180 \text {. }
$$

Two cases are possible. If each subsystem contains eight or more loading complexes, then

$$
\begin{aligned}
& \Delta L=L-28 \geq 4 \times 8-28=4, \\
& \Delta B=B-840 \geq 4 \times 1,600-840=5,560,
\end{aligned}
$$

and by (13.16), (13.17), (13.18), and (8.12), we obtain

$$
\Delta C \geq 374,000 \text {, }
$$

so that in this case the system can be eliminated by comparison with (13.9). The other possible alternative is that each subsystem contains no more than seven loading complexes. Seven loading complexes

13 The same argument, if applied to $M=7$, yields $\Delta C \geq 552,000$, a much stronger result than the one found earlier (the display first above (13.9)). can handle at most

$$
7 \times 36,000=252,000 \text { letters } / \mathrm{hr},
$$

and each of the four subsystems must handle

$$
1,000,000 / 4=250,000 \text { letters } / \mathrm{hr},
$$

so seven complexes/subsystem are needed, all working close to capacity; i.e., the input of each loading complex in a subsystem must be nearly all dropped in the following row of bins in order for the next loading complex to have a nearly empty conveyor belt. This clearly requires at least 200 bins after each of the first 6 complexes in each subsystem; the seventh loader is followed by 1,600 bins (one for each destination) so that

$$
\Delta B=B-840 \geq 4 \times((6 \times 200)+1,600)-840=10,360 ;
$$

from (13.16), (13.19) and (8.12) we obtain

$$
C \geq 390,000,
$$

so that the system is eliminated by comparison with (13.9). Thus systems with $M=4$ are eliminated.

We have not been able, within the limits of time and effort reasonably assignable to this particular point,${ }^{14}$ to devise a mathematical proof that multiple input systems with $M=1,2$, or 3 can be eliminated because of excessive cost. (The difficulties encountered, and the reason for their occurrence for small values of $M$, are discussed in the next paragraph.) Nevertheless, we strongly believe that such systems are excessively costly. This belief is based on auxiliary calculations which will not be reproduced here, and also in general on our experience with the other parts of this study. Roughly, the situation is this: In each subsystem, most of the mail must be dropped out fairly soon after it enters the subsystem, for otherwise the input from many of the loading complexes would be cut substantially below 36,000 letters/hr, so that a large number of loading complexes (whose cost would be excessive) would be required to pass the required $1,000,000$ letters/hour into the system. Such an early dropout of most mail, however, would require a large number of bins, many of which would receive fewer than 60 letters/hr and thus (see (5.3) and (5.4)) involve "inefficient sweeping"; the cost of bins and sweepers (especially the latter) would then be excessive.

The difficulties encountered for small values of $M$ (i.e., for systems with a small number of subsystems) stem from the fact that in such cases each subsystem must contain a relatively large number of loading complexes; for $M=3$, for example, each subsystem contains at least 9 such complexes, while for $M=1$ we have a single long subsystem with at least 28 loading complexes in series. The first cause of difficulty is that the formulas involved in the analysis of a subsystem with $n$ loading complexes

\footnotetext{
14 Especially since the configuration found in section 14 is so nearly optimal.
} 
become more and more complicated as $n$ increases. (See Appendix B for more details.) For $n \geq 9$, for example (i.e., for $M \leq 3$ ), it is still possible to use these formulas in making calculations for any one given subsystem, but is extremely difficult to use them in comparing a large number of possible subsystems; this latter problem is of course, the one which actually arises in our work. The second cause of difficulty is the enormous number of systems to be compared, when the value of $n$ is high (i.e., when $M$ is low); this number is in fact

$$
(n-1)^{\left(2^{d}\right)} \quad(d=\text { number of destinations }),
$$

and since $d=1,600$ it is clear that even if 99 percent of the possible systems could be eliminated on some common-sense grounds, the number remaining for analysis (if $n$ is moderately large) would still be astronomical.

\section{Multiple Input and Residue: Double Loading}

The "multiple input and residue" schemes, combinations of two of the proposed schemes which we have analyzed earlier, are like multiple input schemes except that some of the bins may be assigned to residue; this residue then requires a secondary sort. We therefore speak of "primary subsystems", and "secondary subsystems."

There is a great variety of subclasses of this type of scheme, and within any one subclass the analysis required to determine the optimal choice of the relevant parameters appears to be quite difficult. We will make a detailed analysis only of a relatively simple subclass; the result turns out to be a system so nearly optimal (see (14.25)) that investigation of more complicated systems is clearly not worthwhile.

We consider "multiple input and residue" schemes which are determined by two parameters, $j$ and $k$ (with $j<k$ ), in the following way: Each primary subsystem contains two loading complexes. The first complex of each primary subsystem is followed by bins for each of

the "type 1" destinations $1,2, \ldots, j$,

and also by residue bins for

the "type 3 " destinations $k+1, k+2, \ldots, 1,600$,

Thus that part of the first complex's input consisting of mail to

the "type 2 " destinations $j+1, j+2, \ldots, k$

does not get sorted until after the conveyor passes the second complex. The second complex of each primary subsystem is followed by bins for type 1 and type 2 destinations, and residue bins for the type 3 destinations. We wish to choose $j$ and $k$ so as to minimize the cost of the system.

We will use the notation

$$
f_{i}=\text { fraction of mail to destination } i \text {; }
$$

the destinations are so ordered that

$$
f_{1} \geq f_{2} \geq f_{3} \geq \ldots . \geq f_{1,600} .
$$

We also set

$F_{i}=f_{1}+f_{2}+\ldots+f_{i}=$ fraction of mail to the first $i$ destinations.

The input to the first loading complex of each primary subsystem is the usual 36,000 letters/hr, but only a fraction $F_{j}+\left(1-F_{k}\right)$ of these (corresponding to types 1 and 3 ) get dropped before the second complex, so that the input to the second complex is

$$
36,000\left(1+F_{j}-F_{k}\right) \text { letters } / \mathrm{hr}
$$

and the input to each primary system is

$$
\begin{aligned}
& 36,000+36,000\left(1+F_{j}-F_{k}\right) \\
& =36,000\left(2+F_{j}-F_{k}\right) \text { letters } / \mathrm{hr} \text {. }
\end{aligned}
$$

Therefore the required number of primary subsystems is about

$$
\begin{array}{r}
1,000,000 / 36,000\left(2+F_{j}-F_{k}\right) \\
\approx 28 /\left(2+F_{j}-F_{k}\right),
\end{array}
$$

so that the number of loading complexes for the primary subsystems is about

$$
56 /\left(2+F_{j}-F_{k}\right),
$$

while the residue of $1,000,000\left(1-F_{k}\right)$ letters/hr requires

$$
1,000,000\left(1-F_{k}\right) / 36,000 \approx 28\left(1-F_{k}\right)
$$

loading complexes for secondary sort. Thus

$$
\begin{gathered}
L \approx\left(56 /\left(2+F_{j}-F_{k}\right)\right)+28\left(1-F_{k}\right), \\
\Delta L=L-28 \approx\left(56 /\left(2+F_{j}-F_{k}\right)\right)-28 F_{k}
\end{gathered}
$$

or

The type 3 destinations are divided into groups as for the residue scheme (sec. 12); the number of these groups is given by (14.7). The secondary subsystems together contain one bin for each type 3 destination, or $1,600-k$ bins in all. Each primary subsystem contains $j+k$ bins for separate destinations ( $j$ bins before the second loading complex, $k$ bins after it), and also two residue bins for each group (one before the second complex, Jone after it). Hence, using (14.6) and (14.7), 
$B \approx(1,600-k)+\left(28 /\left(2+F_{j}-F_{k}\right)\right)$

$$
\times\left(j+k+56\left(1-F_{k}\right)\right) \text {, }
$$

so

$$
\begin{aligned}
\Delta B= & B-840 \approx(760-k) \\
& +28\left(j+k+56\left(1-F_{k}\right)\right) /\left(2+F_{j}-F_{k}\right) .
\end{aligned}
$$

Having found $\Delta L$ and $\Delta B$, we still have the more complicated task of finding $\Delta S$. The sweeps of bins of the secondary sort require no extra sweepers. The extra sweepers are required $(a)$ in sweeping the residue bins ${ }^{15}(b)$ in sweeping those bins (if any) before the second loading complex which receive fewer than 60 letters $/ \mathrm{hr}$, and $(c)$ in sweeping those bins after the second complex which receive fewer than 60 letters $/ \mathrm{hr}$. There are

$$
1,000,000\left(1-F_{k}\right) \quad \text { letters/hr }
$$

going to the residue bins, and so ${ }^{15}$ the contribution of (a) to $\Delta S$ is approximately (using (5.1) and (5.2))

$$
1,000,000\left(1-F_{k}\right) /(60 \times 180) .
$$

The analysis of the contribution of $(b)$ to $\Delta S$ depends upon the fact that, for our mail distribution, only destinations 1 to 114 receive 60 or more letters/ hr out of 36,000 letters $/ \mathrm{hr}$ (the input of the first loading complex of a primary subsystem). The contribution of $(b)$ to $\Delta S$ is

$$
0 \quad \text { if } j \leq 114 \text {. }
$$

If $j>114$, then the $j-114$ destinations $115,116, \ldots$., $j$ have their bins before the second loading complex receiving fewer than 60 letters $/ \mathrm{hr}$, leading (via (14.6)) to approximately

$$
28(j-114) /\left(2+F_{j}-F_{k}\right) \text { sweeps/hr. }
$$

Not all of these are extra sweeps, however; the volume of mail involved is ${ }^{16}$

$$
1,000,000\left(F_{j}-F_{114}\right) /\left(2+F_{j}-F_{k}\right) \text { letters } / \mathrm{hr},
$$

which in calculating the minimum cost would receive

$$
1,000,000\left(F_{j}-F_{114}\right) / 60\left(2+F_{j}-F_{k}\right) \text { sweeps } / \mathrm{hr} \text {. }
$$

Thus we have a contribution of $(b)$ to $\Delta S$ of approxi mately

$\left[28(j-114)-1,000,000\left(F_{j}-F_{114}\right) / 60\right] / 180\left(2+F_{j}-F_{k}\right)$ (if $j>114$ ).

(14.12)

The contribution of $(c)$ to $\Delta S$ arises from two sources. First, there are the type 1 destinations (if any) which receive fewer than 60 letters $/ \mathrm{hr}$ after the

\footnotetext{
15 It is assumed that the parameters are so chosen that all residue bins receive at least 60 letters $/ \mathrm{hr}$

${ }_{16}$ For $1 /\left(2+F_{j}-F_{k}\right)$ is the fraction of mail entering the system which enters through the first loading complexes of the primary subsystems.
}

second loading complex of each primary subsystem. To handle these, we define a new variable $m$, dependent on $j$ and $k$, by

destination $m=$ (last destination for which $\left.36,000\left(1+F_{j}-F_{k}\right) f_{m} \geq 60\right)$.

The contribution to $\Delta S$ from the first source of $(c)$ is then

$$
0 \quad \text { if } m>j \text {; }
$$

if $m \leq j$ then the $(j-m)$ destinations $m+1, m+2$, ... $j$ are the ones under consideration, leading via (14.6) to approximately

$$
28(j-m) /\left(2+F_{\jmath}-F_{k}\right)
$$

sweeps, of which (see the derivation of (14.12))

$$
1,000,000\left(F_{j}-F_{m}\right)\left(1+F_{j}-F_{k}\right) / 60\left(2+F_{j}-F_{k}\right)
$$

are extra. Thus we have a contribution to $\Delta S$ from the first source of $(c)$, of approximately

$$
\begin{gathered}
{\left[28(j-m)-1,000,000\left(F_{j}-F m\right)\left(1+F_{j}-F_{k}\right) / 60\right]} \\
\div 180\left(2+F_{j}-F_{k}\right)
\end{gathered}
$$

Second, there are the type 2 destinations which receive fewer than 60 letters/hr after the second complex (i.e., the input from both complexes adds up to fewer than 60 letters $/ \mathrm{hr}$ ). To handle these, we introduce another new variable $n$, also dependent on $j$ and $k$, by

destination $n=$ (last destination for which $\left.36,000\left(2+F_{j}-F_{k}\right) f_{n}>60\right)$.

The contribution to $\Delta S$ from the second source of $(c)$ is then

$$
0 \quad \text { if } n>k \text {; }
$$

reasoning as in the derivations of (14.12) and (14.15) we find the contribution to $\Delta S$ from the second source of $(c)$ to be approximately

$$
\begin{gathered}
{\left[28(k-n)-1,000,000\left(F_{k}-F_{n}\right)\left(2+F_{j}-F_{k}\right) / 60\right]} \\
\div 180\left(2+F_{j}-F_{k}\right) \quad \text { if } j \leq n \leq k
\end{gathered}
$$

and to be approximately

$$
\begin{gathered}
{\left[28(k-j)-1,000,000\left(F_{k}-F_{j}\right)\left(2+F_{j}-F_{k}\right) / 60\right]} \\
\div 180\left(2+F_{j}-F_{k}\right) \quad \text { if } n<j .
\end{gathered}
$$

This completes the derivation of the approximate formula for $\Delta S$.

At this point it is convenient to split $\Delta C$ into two parts,

$$
\Delta C=\Delta_{1} C+\Delta_{2} C,
$$

where $\Delta_{1} C$ represents the effects of $\Delta L, \Delta B$, and the contribution of $(a)$ to $\Delta S$, whereas $\Delta_{2} C$ represents the effects of the contributions of $(b)$ and $(c)$ to $\Delta S$. 
By (8.12) and (14.10),

$$
\begin{aligned}
\Delta_{1} C & \approx 12,700(\Delta L)+14(\Delta B) \\
& +14,670\left(1,000,000\left(1-F_{k}\right) /(60)(180)\right),
\end{aligned}
$$

which, together with (14.8) and (14.9), yields

$$
\begin{aligned}
\Delta_{1} C & \approx\left[37.48 \times 10^{9}+\left(4.234 \times 10^{6}\right) j+\left(3.931 \times 10^{6}\right) k\right. \\
& +\left(14.78 \times 10^{9}\right) F_{j}-\left(52.04 \times 10^{9}\right) F_{k} \\
& \left.+\left(18.51 \times 10^{9}\right)\left(F_{k}^{2}-F_{j} F_{k}\right)\right] /(60)(180) \times \\
& \left(2+F_{j}-F_{k}\right),
\end{aligned}
$$

where the lower-order-of-magnitude quantity

$$
\left(1.512 \times 10^{5}\right) k\left(F_{k}-F_{j}\right)
$$

has been dropped from the numerator of (14.22). No single formula can be given for $\Delta_{2} C$. This is because $\Delta_{2} C$ is the sum of three terms, the first of which is obtained (by multiplication by 14,670) from either (14.11) or (14.12) (according as $j \leq 114$ or $j>114$ ), the second of which is obtained (by multiplication by 14,670) from either (14.14) or (14.15) (according as $m>j$ or $m \leq j$ ), from the third of which is obtained (by multiplication by 14,670 ) from either (14.17) or (14.18) or (14.19) (according as $n>k$ or $j \leq n \leq k$ or $n<j)$. These diverse possibilities lead to $2 \times 2 \times 3=12$ cases; the case-by-case analysis is quite complicated, and we relegate it to appendix $\mathrm{C}$, giving here only the result:

The cost $\Delta C$ is approximately minimized for our particular mail distribution by choosing

$$
j=120, \quad k=264,
$$

yielding

$$
\Delta C \approx 366,000
$$

so that

$$
\left(C-C_{\min }^{*} / C_{\min }^{*}\right) \approx 4 \% .
$$

The minimum is a rather insensitive one; if $j$ is chosen anywhere between roughly 90 and 150 then (assuming $k$ is properly chosen) $\left(C-C_{\min }^{*}\right) / C_{\min }^{*}$ will be less than 0.5 percent above its minimum.

The analysis given above (and continued in app. C) has employed the "add up" method only, deliberately avoiding any use of the "follow through" approach of section 9 . We conclude this section by showing how the "follow through" method can be used (i) to reduce substantially the calculations of appendix C, and (ii) to provide rather good approximations to the optimal $(j, k)$-pair, (14.23). Our argument will show that $j$ and $k$ should be chosen to obey

$$
j \geq 105, \quad k \leq 281 .
$$

Use of this information would have permitted significantly less work $^{17}$ in treating cases 8 to 12 (the most difficult cases) in appendix C. Furthermore, if we regard (14.26) as suggesting $j=105, \quad k=$ 281 as an approximation to an optimal choice, we find that for these values $\left(C-C_{\min }^{*}\right) / C_{\min }^{*}$ is less

17 It would also yield a quick elimination of cases 1 to 5 in appendix C. than 0.2 percent above the value determined by (14.23).

We recall the definitions (14.1) to (14.3) of the three types of destinations. Our first assertion is that:

if $10^{6} f_{i} \leq 863$, then the $i$ th destination should not be a type 1 destination

(14.27)

For, we found in the "simple" residue scheme of section 12 that if $10^{6} f_{i} \leq 863$ then mail to the $i$ th destination should go into residue rather than receive its final sort in the primary. Since this applied after one loading complex operating at full capacity (and thus applies after the first complex of each of our primary subsystems here), there is even more reason for it to apply after a loading complex operating below capacity (such as the second complex in each of our primary subsystems). Thus it would be better to have the $i$ th destination as type 3 than as type 1, and so (14.27) is proved. Of course, it might be still better to have the $i$ th destination in type 2 , and this is the next question to be considered.

If the $i$ th destination is taken to be type 2 , then (according to (9.14) and (9.17)) the cost associated with a letter to it would be denoted $C\left(f_{i}, 2\right)$. The formula (9.18) for $C\left(f_{i}, n_{i}\right)$ was derived assuming all primary loading complexes operating at capacity; this formula therefore provides a lower bound for $C\left(f_{i}, n_{i}\right)$ in our actual system, so that the special case $(9.25)$ of $(9.18)$ yields

$$
C\left(f_{i}, 2\right) \geq 0.5295+\left(1.337 \times 10^{-3} / f_{i}\right)\left(\text { if } 10^{6} f_{i} \leq 840\right) .
$$

If, on the other hand, the $i$ th destination is taken to be type 3 , then (according to (9.11)) the appropriate cost is denoted $C\left(f_{i}\right)$. Except for a negligible correction due to possible violation of $(9.2)$, the formula (9.12) still applies, yielding as in (9.24)

$$
C\left(f_{i}\right)=3.434+\left(14 / 10^{6} f_{i}\right) \quad\left(\text { if } 10^{6} f_{i} \geq 60\right) .
$$

From (14.28 and (14.29) we find that

$$
C\left(f_{i}, 2\right)-C\left(f_{i}\right) \geq 0 \text { for } 60 \leq 10^{6} f_{i} \leq 455 ;
$$

i.e., if $10^{6} f_{i} \leq 455$, then the $i$ th destination should be taken as type 3 rather than type 2 .

(14.30)

From (14.30) we have $10^{6} f_{k}>455$, which for our particular mail distribution yields $k \leq 281$.

Next we recall that in the discussion in section 9 (see (9.46)) the change from $\left(n_{i}\right)_{\mathrm{opt}}=2$ to $\left(n_{i}\right)_{\mathrm{opt}}=1$ occurred for $10^{6} f_{i}=1,614$; that is, for $10^{6} f_{i} \geq 1,614$ it was better to sort a letter to the $i$ th destination directly after it enters the system, rather than either to put it into residue or to send it on past another loader complex. In our current situation this condition must be altered to take account of the fact that the second loading complex of each subsystem operates at only a fraction $1+F_{j}-F_{k}$ of capacity. 
We find thus that for

$$
\left(1+F_{j}-F_{k}\right) 10^{6} f_{i} \geq 1,614 \quad\left(10^{6} f_{i} \geq 864\right)
$$

on the basis of input to the second loading complexes only, the $i$ th destination should be of type 1 rather than type 2 . Since $k \leq 281$ implies $F_{k} \leq F_{281}$, it follows that the $j \geq 105$. Thus (14.26) is obtained. $i$ th destination should be of type 1 rather than type 2 if

$$
\left(1+F_{j}-F_{281}\right) 10^{6} f_{i} \geq 1,614,
$$

so that in particular we should have

$$
\left(1+F_{j}-F_{281}\right) 10^{6} f_{j+1}<1,614,
$$

which for our particular mail distribution yields

\section{Appendix A: Mail Distribution by Destinations}

\begin{tabular}{|c|c|c|c|c|c|c|c|c|c|c|c|}
\hline$i$ & $f_{i}$ & $F_{i}$ & $i$ & $f_{i}$ & $F_{i}$ & $i$ & $f_{i}$ & $F_{i}$ & $i$ & $f_{i}$ & $F_{i}$ \\
\hline $\begin{array}{l}1 \\
2 \\
3 \\
4 \\
5\end{array}$ & $\begin{array}{l}\text { \% } \\
3.912 \\
2.967 \\
2.893 \\
2.626 \\
2.480\end{array}$ & $\begin{array}{r}\% \\
3.912 \\
6.879 \\
9.772 \\
12.398 \\
14.878\end{array}$ & $\begin{array}{l}56 \\
57 \\
58 \\
59 \\
60\end{array}$ & $\begin{array}{ll}\% & \\
.375 \\
.371 \\
.366 \\
.366 \\
.353\end{array}$ & $\begin{array}{l}\%^{*} \\
57.659 \\
58.030 \\
58.396 \\
58.762 \\
59.115\end{array}$ & $\begin{array}{l}111 \\
112 \\
113 \\
114 \\
115\end{array}$ & $\begin{array}{r} \\
.172 \\
.172 \\
.170 \\
.170 \\
.166\end{array}$ & $\begin{array}{l}\% \\
71.887 \\
72.059 \\
72.229 \\
72.399 \\
72.565\end{array}$ & $\begin{array}{l}166 \\
167 \\
168 \\
169 \\
170\end{array}$ & $\begin{array}{r}\% \\
.110 \\
.110 \\
.108 \\
.108 \\
.106\end{array}$ & $\begin{array}{l}\% \\
79.471 \\
79.581 \\
79.689 \\
79.797 \\
79.903\end{array}$ \\
\hline $\begin{array}{r}6 \\
7 \\
8 \\
9 \\
10\end{array}$ & $\begin{array}{l}2.230 \\
2.057 \\
2.044 \\
\text { 1. } 754 \\
1.648\end{array}$ & $\begin{array}{l}17.108 \\
19.165 \\
21.209 \\
22.963 \\
24.611\end{array}$ & $\begin{array}{l}61 \\
62 \\
63 \\
64 \\
65\end{array}$ & $\begin{array}{l}.345 \\
.345 \\
.343 \\
.319 \\
.319\end{array}$ & $\begin{array}{l}59.460 \\
59.805 \\
60.148 \\
60.467 \\
60.786\end{array}$ & $\begin{array}{l}116 \\
117 \\
118 \\
119 \\
120\end{array}$ & $\begin{array}{l}.166 \\
.166 \\
.166 \\
.164 \\
.157\end{array}$ & $\begin{array}{l}72.731 \\
72.897 \\
73.063 \\
73.227 \\
73.384\end{array}$ & $\begin{array}{l}171 \\
172 \\
173 \\
174 \\
175\end{array}$ & $\begin{array}{l}.106 \\
.106 \\
.106 \\
.101 \\
.101\end{array}$ & $\begin{array}{l}80.009 \\
80.115 \\
80.221 \\
80.322 \\
80.423\end{array}$ \\
\hline $\begin{array}{l}11 \\
12 \\
13 \\
14 \\
15\end{array}$ & $\begin{array}{l}1.635 \\
1.622 \\
1.504 \\
1.467 \\
1.379\end{array}$ & $\begin{array}{l}26.246 \\
27.868 \\
29.372 \\
30.839 \\
32.218\end{array}$ & $\begin{array}{l}66 \\
67 \\
68 \\
69 \\
70\end{array}$ & $\begin{array}{l}.319 \\
.317 \\
.317 \\
.315 \\
.312\end{array}$ & $\begin{array}{l}61.105 \\
61.422 \\
61.739 \\
62.054 \\
62.366\end{array}$ & $\begin{array}{l}121 \\
122 \\
123 \\
124 \\
125\end{array}$ & $\begin{array}{l}.154 \\
.154 \\
.154 \\
.152 \\
.152\end{array}$ & $\begin{array}{l}73.538 \\
73.692 \\
73.846 \\
73.998 \\
74.150\end{array}$ & $\begin{array}{l}176 \\
177 \\
178 \\
179 \\
180\end{array}$ & $\begin{array}{l}.101 \\
.101 \\
.099 \\
.097 \\
.097\end{array}$ & $\begin{array}{l}80.524 \\
80.625 \\
80.724 \\
80.821 \\
80.918\end{array}$ \\
\hline $\begin{array}{l}16 \\
17 \\
18 \\
19 \\
20\end{array}$ & $\begin{array}{r}1.256 \\
1.163 \\
1.073 \\
1.053 \\
.982\end{array}$ & $\begin{array}{l}33.474 \\
34.637 \\
35.710 \\
36.763 \\
37.745\end{array}$ & $\begin{array}{l}71 \\
72 \\
73 \\
74 \\
75\end{array}$ & $\begin{array}{l}.308 \\
.302 \\
.297 \\
.289 \\
.287\end{array}$ & $\begin{array}{l}62.674 \\
62.976 \\
63.273 \\
63.562 \\
63.849\end{array}$ & $\begin{array}{l}126 \\
127 \\
128 \\
129 \\
130\end{array}$ & $\begin{array}{l}.152 \\
.150 \\
.150 \\
.150 \\
.147\end{array}$ & $\begin{array}{l}\text { 74. } 302 \\
74.452 \\
74.602 \\
74.752 \\
74.899\end{array}$ & $\begin{array}{l}181 \\
182 \\
183 \\
184 \\
185\end{array}$ & $\begin{array}{l}.095 \\
.095 \\
.095 \\
.095 \\
.095\end{array}$ & $\begin{array}{l}81.013 \\
81.108 \\
81.203 \\
81.298 \\
81.393\end{array}$ \\
\hline $\begin{array}{l}21 \\
22 \\
23 \\
24 \\
25\end{array}$ & $\begin{array}{l}.827 \\
.814 \\
.773 \\
.734 \\
.713\end{array}$ & $\begin{array}{l}38.572 \\
39.386 \\
40.159 \\
40.893 \\
41.606\end{array}$ & $\begin{array}{l}76 \\
77 \\
78 \\
79 \\
80\end{array}$ & $\begin{array}{l}.278 \\
.273 \\
.271 \\
.267 \\
.265\end{array}$ & $\begin{array}{l}64.127 \\
64.400 \\
64.671 \\
64.938 \\
65.203\end{array}$ & $\begin{array}{l}131 \\
132 \\
133 \\
134 \\
135\end{array}$ & $\begin{array}{l}.145 \\
.145 \\
.140 \\
.140 \\
.140\end{array}$ & $\begin{array}{l}75.044 \\
75.189 \\
75.329 \\
75.469 \\
75.609\end{array}$ & $\begin{array}{l}186 \\
187 \\
188 \\
189 \\
190\end{array}$ & $\begin{array}{l}.095 \\
.093 \\
.091 \\
.091 \\
.088\end{array}$ & $\begin{array}{l}81.488 \\
81.581 \\
81.672 \\
81.763 \\
81.851\end{array}$ \\
\hline $\begin{array}{l}26 \\
27 \\
28 \\
29 \\
30\end{array}$ & $\begin{array}{l}.706 \\
.702 \\
.655 \\
.644 \\
.623\end{array}$ & $\begin{array}{l}\text { 42. } 312 \\
\text { 43. } 014 \\
\text { 43. } 669 \\
\text { 44. } 313 \\
\text { 44. } 936\end{array}$ & $\begin{array}{l}81 \\
82 \\
83 \\
84 \\
85\end{array}$ & $\begin{array}{l}.265 \\
.260 \\
.260 \\
.254 \\
.252\end{array}$ & $\begin{array}{l}65.468 \\
65.728 \\
65.988 \\
66.242 \\
66.494\end{array}$ & $\begin{array}{l}136 \\
137 \\
138 \\
139 \\
140\end{array}$ & $\begin{array}{l}.138 \\
.138 \\
.138 \\
.138 \\
.136\end{array}$ & $\begin{array}{l}75.747 \\
75.885 \\
76.023 \\
76.161 \\
76.297\end{array}$ & $\begin{array}{l}191 \\
192 \\
193 \\
194 \\
195\end{array}$ & $\begin{array}{l}.088 \\
.088 \\
.088 \\
.088 \\
.088\end{array}$ & $\begin{array}{l}81.939 \\
82.027 \\
82.115 \\
82.203 \\
82.291\end{array}$ \\
\hline $\begin{array}{l}31 \\
32 \\
33 \\
34 \\
35\end{array}$ & $\begin{array}{l}.615 \\
.604 \\
.580 \\
.571 \\
.551\end{array}$ & $\begin{array}{l}45.551 \\
46.155 \\
46.735 \\
47.306 \\
47.857\end{array}$ & $\begin{array}{l}86 \\
87 \\
88 \\
89 \\
90\end{array}$ & $\begin{array}{l}.246 \\
.246 \\
239 \\
.233 \\
.233\end{array}$ & $\begin{array}{l}66.740 \\
66.986 \\
67.225 \\
67.458 \\
67.691\end{array}$ & $\begin{array}{l}141 \\
142 \\
143 \\
144 \\
145\end{array}$ & $\begin{array}{l}.136 \\
.136 \\
.136 \\
.134 \\
.134\end{array}$ & $\begin{array}{l}76.433 \\
76.569 \\
76.705 \\
76.839 \\
76.973\end{array}$ & $\begin{array}{l}196 \\
197 \\
198 \\
199 \\
200\end{array}$ & $\begin{array}{l}.088 \\
.086 \\
.086 \\
.084 \\
.082\end{array}$ & $\begin{array}{l}82.379 \\
82.465 \\
82.551 \\
82.635 \\
82.717\end{array}$ \\
\hline $\begin{array}{l}36 \\
37 \\
38 \\
39 \\
40\end{array}$ & $\begin{array}{l}.538 \\
.534 \\
.534 \\
.534 \\
.508\end{array}$ & $\begin{array}{l}48.395 \\
48.929 \\
49.463 \\
49.997 \\
50.505\end{array}$ & $\begin{array}{l}91 \\
92 \\
93 \\
94 \\
95\end{array}$ & $\begin{array}{l}.224 \\
.224 \\
.220 \\
.218 \\
.214\end{array}$ & $\begin{array}{l}67.915 \\
68.139 \\
68.359 \\
68.577 \\
68.791\end{array}$ & $\begin{array}{l}146 \\
147 \\
148 \\
149 \\
150\end{array}$ & $\begin{array}{l}.132 \\
.132 \\
.130 \\
.128 \\
.126\end{array}$ & $\begin{array}{l}77.105 \\
77.237 \\
77.367 \\
77.495 \\
77.621\end{array}$ & $\begin{array}{l}201 \\
202 \\
203 \\
204 \\
205\end{array}$ & $\begin{array}{l}.080 \\
.080 \\
.078 \\
.078 \\
.076\end{array}$ & $\begin{array}{l}82.797 \\
82.877 \\
82.955 \\
83.033 \\
83.109\end{array}$ \\
\hline $\begin{array}{l}41 \\
42 \\
43 \\
44 \\
45\end{array}$ & $\begin{array}{l}.508 \\
.506 \\
.501 \\
.491 \\
.491\end{array}$ & $\begin{array}{l}51.013 \\
51.519 \\
52.020 \\
52.511 \\
53.002\end{array}$ & $\begin{array}{r}96 \\
97 \\
98 \\
99 \\
100\end{array}$ & $\begin{array}{l}.211 \\
.211 \\
.209 \\
.202 \\
.200\end{array}$ & $\begin{array}{l}69.002 \\
69.213 \\
69.422 \\
69.624 \\
69.824\end{array}$ & $\begin{array}{l}151 \\
152 \\
153 \\
154 \\
155\end{array}$ & $\begin{array}{l}.126 \\
.126 \\
.122 \\
.116 \\
.116\end{array}$ & $\begin{array}{l}77.747 \\
77.873 \\
77.995 \\
78.111 \\
78.227\end{array}$ & $\begin{array}{l}206 \\
207 \\
208 \\
209 \\
210\end{array}$ & $\begin{array}{l}.076 \\
.076 \\
.074 \\
.074 \\
.074\end{array}$ & $\begin{array}{l}83.185 \\
83.261 \\
83.335 \\
83.409 \\
83.483\end{array}$ \\
\hline $\begin{array}{l}46 \\
47 \\
48 \\
49 \\
50\end{array}$ & $\begin{array}{l}.483 \\
.465 \\
.461 \\
.457 \\
.445\end{array}$ & $\begin{array}{l}53.485 \\
53.950 \\
54.411 \\
54.868 \\
55.313\end{array}$ & $\begin{array}{l}101 \\
102 \\
103 \\
104 \\
105\end{array}$ & $\begin{array}{l}.198 \\
.198 \\
.196 \\
.196 \\
.192\end{array}$ & $\begin{array}{l}70.022 \\
70.220 \\
70.416 \\
70.612 \\
70.804\end{array}$ & $\begin{array}{l}156 \\
157 \\
158 \\
159 \\
160\end{array}$ & $\begin{array}{l}.116 \\
.114 \\
.114 \\
.114 \\
.114\end{array}$ & $\begin{array}{l}78.343 \\
78.457 \\
78.571 \\
78.685 \\
78.799\end{array}$ & $\begin{array}{l}211 \\
212 \\
213 \\
214 \\
215\end{array}$ & $\begin{array}{l}.073 \\
.073 \\
.073 \\
.071 \\
.071\end{array}$ & $\begin{array}{l}83.556 \\
83.629 \\
83.702 \\
83.773 \\
83.844\end{array}$ \\
\hline $\begin{array}{l}51 \\
52 \\
53 \\
54 \\
55\end{array}$ & $\begin{array}{l}.407 \\
.401 \\
.394 \\
.390 \\
.379\end{array}$ & $\begin{array}{l}55.720 \\
56.121 \\
56.515 \\
56.905 \\
57.284\end{array}$ & $\begin{array}{l}106 \\
107 \\
108 \\
109 \\
110\end{array}$ & $\begin{array}{l}.187 \\
.187 \\
.185 \\
.177 \\
.175\end{array}$ & $\begin{array}{l}70.991 \\
71.178 \\
71.363 \\
71.540 \\
71.715\end{array}$ & $\begin{array}{l}161 \\
162 \\
163 \\
164 \\
165\end{array}$ & $\begin{array}{l}.114 \\
.114 \\
.112 \\
.112 \\
.110\end{array}$ & $\begin{array}{l}78.913 \\
79.027 \\
79.139 \\
79.251 \\
79.361\end{array}$ & $\begin{array}{l}216 \\
217 \\
218 \\
219 \\
220\end{array}$ & $\begin{array}{l}.070 \\
.070 \\
.070 \\
.068 \\
.068\end{array}$ & $\begin{array}{l}83.914 \\
83.984 \\
84.054 \\
84.122 \\
84.190\end{array}$ \\
\hline
\end{tabular}

In the following table $f_{i}$ denotes the fraction of mail to the $i$ th destination, and $F_{i}$ denotes the fraction of mail to the first $i$ destinations so that $F_{i}=f_{1}+f_{2}+\ldots+f_{i-1}+f_{i}$ 
$F_{i}=f_{1}+f_{2}+\ldots+f_{i-1}+f_{i}$.

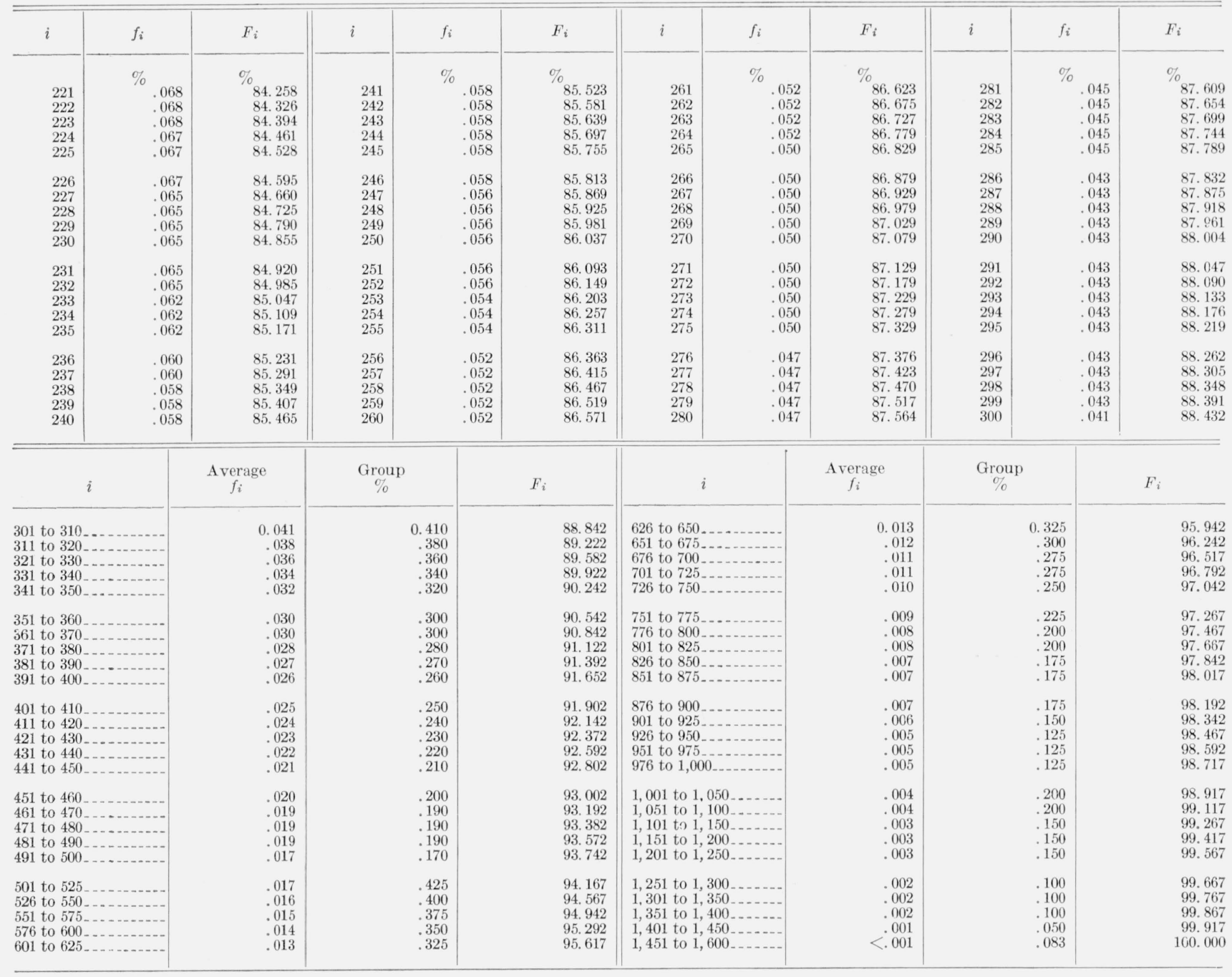

\section{Appendix B: Analysis of Multiple Input Systems}

We consider a multiple input system in which

$$
\begin{aligned}
M= & \text { number of subsystems } \\
n= & \text { number of loading complexes in each } \\
& \text { subsystem. }
\end{aligned}
$$

In order to specify the system completely, we must also specify, for $i=1,2, \ldots, n$

$\begin{aligned} D_{i}= & \text { set of destinations whose letters are dropped } \\ & \text { in the row of bins after the } i \text { th loading com- } \\ & \text { plex in each subsystem. }\end{aligned}$
( $D_{n}$ will necessarily consist of all the destinations.) The sets $D_{1}, \ldots$, $D_{n}$ may overlap (for example, some destinations may be included both in $D_{1}$ and in $D_{2}$ ), so that it is also necessary to deal with combinations of the $D_{i}$ 's such as $D_{1}-D_{2}$ (the set of destinations included in $D_{1}$ but not in $D_{2}$ ). If $\mathscr{D}$ is any set of destinations (for example, $\mathscr{D}$ might be $D_{1}$ or $\left.D_{1}-D_{2}\right)$, we use the notation

$$
\begin{gathered}
F(\mathscr{D})=\text { fraction of the total mail which } \\
\text { goes to destinations in } \mathscr{D} \text {. }
\end{gathered}
$$

We wish now to analyze the inputs to the various loading complexes, and also the input to each subsystem as a whole. Let 
$I_{i}=$ ratio of the actual input to the $i$ th loading complex of each subsystem to its maximum possible input of 36,000 letters/hr.

(B5)

The quantities $I_{1}, I_{2}, \ldots, I_{n}$ (and thus the inputs $\left.36,000 I_{1}, 36,000 I_{2}, \ldots, 36,000 I_{n}\right)$ can be computed one by one from the formulas

$I_{1}=1$

$I_{2}=F\left(D_{1}\right)$,

$I_{3}=F\left(D_{2}-D_{1}\right)+I_{2} F\left(D_{2}\right)$,

$I_{4}=F\left(D_{3}-D_{2}-D_{1}\right)+I_{2} F\left(D_{3}-D_{2}\right)+I_{3} F\left(D_{3}\right)$,

$$
\begin{aligned}
I_{n}=F & \left(D_{n-1}-D_{n-2}-\ldots-D_{1}\right) \\
& +I_{2} F\left(D_{n-1}-D_{n-2} \ldots-D_{2}\right) \\
& +\ldots+I_{n-1} F\left(D_{n-1}\right) .
\end{aligned}
$$

To obtain a more compact notation for these equations, we adopt the convention $I_{0}=0$ and define the sets of destinations

$D_{i j}^{*}=D_{i-1}-D_{i-2}-\ldots-D_{j} \quad(j<i-1)$;

then (B6) can be rewritten as

$$
I_{i}=\sum_{j<i} F\left(D_{i j}^{*}\right) I_{j} .
$$

The input to each subsystem is given by

$$
\text { (input/subsystem) }=36,000\left(I_{1}+I_{2}+\ldots+I_{n}\right) .
$$

\section{Appendix C: Proofs of Results Asserted in Section 14}

The following material presupposes familiarity with section 14, to which frequent reference is made. We recall that $\Delta C$ had been split into two parts,

$$
\Delta C=\Delta_{1} C+\Delta_{2} C,
$$

that a formula (14.22) had been derived for $\Delta_{1} C$, but that $\Delta_{2} C$ could apparently be given by any one of 12 possible formulas, leading to 12 possible cases requiring analysis.

First we write down the conditions defining the 5 cases (out of these 12) which can be treated most easily:

$\begin{array}{llll}\text { Case 1: } & j \leq 114, & m>j, & n<j . \\ \text { Case 2: } & j>114, & m>j, & n<j . \\ \text { Case 3: } & j \leq 114, & m \leq j, & n<j . \\ \text { Case 4: } & j>114, & m>j, & n>k . \\ \text { Case 5: } & j>114, & m>j, & j \leq n \leq k\end{array}$

Case 5: $j>114, \quad m>j, \quad j \leq n \leq k$.
These cases will be proved logically impossible. From (14.13) and (14.16) it can be deduced that $f_{m}>f_{n}$, so that $m<n$, ruling out cases 1 and 2. The first and third conditions of case 3 would yield, using (14.16),

$$
1 / 600 \approx f_{114} \leq f_{j} \leq f_{n} \approx 1 / 600\left(2+F_{j}-F_{k}\right),
$$

which is impossible since

$$
2+F_{j}-F_{k} \geq 2+F_{j}-1 \geq 1+F_{114}=1.724 ;
$$

thus case 3 is ruled out. The first and second conditions common to cases 4 and 5 yield, using (14.13),

$$
1 / 600 \approx f_{114}>f_{j} \geq f_{m} \approx 1 / 600\left(1+F_{j}-F_{k}\right),
$$

which is impossible since (using $j \leq k$ to deduce $\left.F_{j} \leq F_{k}\right)$ we have

$$
1+F_{j}-F_{k} \leq 1+F_{k}-F_{k}=1 ;
$$

thus cases 4 and 5 are ruled out.

Next we describe the technique to be used in handling some of the remaining seven cases. The parameters $j$ and $k$ will be treated as continuous (rather than integer-valued) variables, so that calculus methods can be used in searching for the minimum of $\Delta C$. As the two independent variables, it is convenient to choose not $j$ and $k$, but rather

$$
j \quad \text { and } \quad u=1+F_{j}-F_{k} .
$$

Then $k$ becomes a dependent variable, and from (C1) we have

so that

$$
\partial F_{k} / \partial j=f_{j},
$$

$$
\partial k / \partial j=\left(\partial F_{k} / \partial j\right) /\left(d F_{k} / d k\right)=f_{j} / f_{k} .
$$

For our purposes, it is sufficiently accurate to replace (14.13) and (14.16) by

$$
f_{m}=1 / 600 u, \quad f_{n}=1 / 600(1+u),
$$

so $f_{m}$ and $f_{n}$ (hence $m, n, F_{m}$, and $F_{n}$ ) depend only on $u$ and not on $j$, yielding

$$
\partial m / \partial j=\partial n / \partial j=\partial F_{m} / \partial j=\partial F_{n} / \partial j=0
$$

A necessary condition for a minimum of $\Delta C$ is

$$
\partial(\Delta C) / \partial j=0,
$$

and if we introduce the symbol $D$ solely as an abbreviation for the frequently-occurring quantity

$$
D=f_{k}(1+u) \partial(\Delta C) / \partial j,
$$

then it follows that a necessary condition for a minimum of $\Delta C$ is

$$
D=0
$$

We will be able to eliminate a number of the remaining seven cases by showing that they are incompatible with $(\mathbf{C} 7)$. 
A useful preliminary step is obtained by rewriting (14.8) as

$$
\Delta L=-28\left(1+F_{j}-u\right)+56 /(1+u),
$$

rewriting (14.9) as

$$
\Delta B=(760-k)+28\left(j+k+56\left(u-F_{j}\right)\right) /(1+u),
$$

noting that (14.10) can be written as

$$
10^{6}\left(u-F_{j}\right) / 60 \times 180
$$

and then using (14.22) and (C1) to (C3) to obtain

$$
\begin{aligned}
f_{k}(1+u) \partial\left(\Delta_{1} C\right) / \partial j & =-17.139 \times 10^{5} f_{j} f_{k}(1+u) \\
& -14 f_{j}(1+u)-2.195 \times 10^{4} f_{j} f_{k}+392\left(f_{j}+f_{k}\right) .
\end{aligned}
$$

The next 3 cases (out of the remaining 7 ) to be treated are

Case 6: $j \leq 114, \quad m>j, \quad n>k$

Case 7: $\quad j>114, \quad m \leq j, \quad n<j$

Case 8: $j \leq 114, \quad m \leq j, \quad n>k$

These cases are logically possible, but we will prove that they do not give rise to the minimum of $\Delta C$.

In case $6, \Delta C=\Delta_{1} C$, so that by $(\mathrm{C} 6)$ and $(\mathrm{C} 8)$,

$$
\begin{aligned}
D=-17.139 \times 10^{5} f_{j} f_{k}(1+u)-14 f_{j}(1+u) \\
-2.195 \times 10^{4} f_{j} f_{k}+392\left(f_{j}+f_{k}\right) .
\end{aligned}
$$

Since (C4) and the last condition of case 6 imply

$$
f_{k}(1+u)>1 / 600
$$

we can deduce from (C9), using the fact $f_{j} \geq f_{k}$, that $\mathrm{D}<-17.139 \times 10^{5} f_{j} f_{k}(1+u)+784 f_{j}$

$$
<\left(-17.139 \times 10^{5} / 600\right) f_{j}+784 f_{j}<0,
$$

so that in case $6, D<0$, contradicting $(\mathrm{C} 7)$.

In case $7, \Delta C$ is obtained by adding to $\Delta_{1} C$ the sum (multiplied by 14,670$)$ of $(14.12),(14.15)$, and (14.19). Using (C1) to (C6), we obtain

$$
\begin{array}{r}
D=-30.722 \times 10^{5} f_{j} f_{k}(1+u)-14 f_{j} u-.22 \times 10^{5} f_{j} f_{k} \\
+2,660 f_{j}+2,674 f_{k} .
\end{array}
$$

Since the third condition of case 7 , together with (C4), implies

$$
f_{j}(1+u)<1 / 600,
$$

while the first condition (together with $j \leq k$ ) implies

we have

$$
f_{k} \leq f_{j} \leq f_{115}<1 / 110
$$

$$
\begin{aligned}
D> & \left(-30.722 \times 10^{5} / 600\right) f_{k}-22,000 f_{i} f_{k}+2,646 f_{j} \\
+2,674 f_{k}=2,646 f_{j}-2,446 f_{k}-22,000 f_{j} f_{k}>200 f_{j} & \\
-22,000 f_{j} f_{k}=200 f_{j}\left(1-110 f_{k}\right) & >0,
\end{aligned}
$$

so that in case $7, D>0$, contradicting $(\mathrm{C} 7)$.

In case $8, \Delta C$ is obtained by adding to $\Delta_{1} C$ the product of (14.15) by 14,670. Using (C1) to (C6), we obtain

$D=-30.722 \times 10^{5} f_{i} f_{k}(1+u)+13.363 \times 10^{5} f_{i} f_{k}$

$$
+378 f_{j}+2,674 f_{k}-14 f_{j} u \text {. }
$$

The third condition of case 8 (together with (C4)) implies

$$
f_{k}(1+u)>1 / 600,
$$

so that (using the fact that $f_{k} \leq f_{j}$ )

$$
\begin{aligned}
D<\left(-30.722 \times 10^{5} / 600\right) f_{j} & +13.363 \times 10^{5} f_{j} f_{k}+3,052 f_{j} \\
& =f_{j}\left(13.363 \times 10^{5} f_{k}-2,068\right) .
\end{aligned}
$$

Thus $D<0$ if $13.363 \times 10^{5} f_{k}-2.068<0$ : i.e., if $f_{k}<.00155$, or equivalently, if $k>120$. Hence, in the remainder of the discussion of case 8 , we can ssume that $k \leq 120$, so that $F_{k} \leq F_{120}$. The second condition of case 8, together with (C4), yields.

$$
1 / 600>f_{j}\left(1+F_{j}-F_{k}\right),
$$

so that, using the fact $F_{k} \leq F_{120}$, we obtain

$$
1 / 600>f_{j}\left(.2662+F_{j}\right) .
$$

By the first condition of case $8, f_{j} \geq f_{114}$, so that by (C10),

$$
1 / 600>f_{114}\left(.2262+F_{j}\right),
$$

or $\quad F_{j} \leq .7142, \quad$ implying $\quad j \leq 108$,

so by $\left(\right.$ C10 $1 / 600>f_{108}\left(.2262+F_{j}\right)$

or $\quad F_{j} \leq .6347 \quad$ implying $j \leq 73$,

so by $(\mathrm{C} 10) \quad 1 / 600>f_{73}\left(.2262+F_{j}\right)$

or $\quad F_{j} \leq .2949 \quad$ implying $\quad j \leq 13$,

so by $(\mathrm{C} 10) \quad 1 / 600>f_{13}\left(.2262+F_{j}\right)$,

or

$$
F_{j} \leq-0.1552,
$$

which is impossible since $F_{j} \geq 0$. Thus case 8 is eliminated.

There are now only four cases left. We now examine

Case 9: $j \leq 114, \quad m \leq j, \quad j \leq n \leq k$.

It turns out that $D=0$ can occur in this case, but only in the subcase defined by

$$
99 \leq j \leq 114, \quad 187 \leq k \leq 281 .
$$

To prove this, we note first that in case $9, \Delta C$ is obtained by adding to $\Delta_{1} C$ the sum (multiplied by 
14,670) of (14.15) and (14.18); using (C1) to (C6), this yields

$$
\begin{aligned}
D=-44.305 \times 10^{5} f_{j} f_{k} u-14 f_{j} u- & 30.942 \times 10^{5} f_{j} f_{k} \\
& +2,660 f_{j}+2,674 f_{k} .
\end{aligned}
$$

By the second condition of case 9 (together with (C4)),

$$
f_{j} u<1 / 600,
$$

which together with $u \leq 1$ yields

$$
\begin{array}{r}
D>\left(-44.305 \times 10^{5} / 600\right) f_{k}-30.942 \times 10^{5} f_{j} f_{k}+2,646 f_{j} \\
+2,674 f_{k}=2,646 f_{j}-f_{k}\left(30.942 \times 10^{5} f_{j}+4,710\right),
\end{array}
$$

so that $D>0$ if

$$
f_{k} \leq 2,646 f_{j} /\left(4,710+30.942 \times 10^{5} f_{j}\right)
$$

or equivalently, if

$$
f_{k} \leq 1 /\left(1,169.4+1.780 / f_{j}\right) .
$$

Thus in the remainder of the discussion of case 9 , we can assume that

$$
f_{k}>1 /\left(1,169.4+1.780 / f_{j}\right) .
$$

From the first condition of case 9 we have $f_{j} \geq f_{114}$, and combining this with the last inequality yields

$$
f_{k}>.00045
$$

so that $k \leq 281$ as asserted in (C11). Thus,

$$
F_{k} \leq F_{281}
$$

which together with the version

$$
1 / 600>f_{j}\left(1+F_{j}-F_{k}\right)
$$

of (C12) yields $1 / 600>f_{j}\left(1+F_{j}-F_{281}\right)$, or

$$
1 / 600>f_{j}\left(.12391+F_{j}\right) \text {. }
$$

Since $j \geq 1$, we have $F_{j} \geq F_{1}$ so that by (C13)

or

$$
1 / 600>f_{j}\left(.12391+F_{1}\right),
$$

$$
f_{j}<.0102 \quad \text { implying } \quad j \geq 20,
$$

so by (C13)

$$
1 / 600>f_{j}\left(.12391+F_{20}\right),
$$

or

$$
f_{j}<.00332 \quad \text { implying } \quad j \geq 64,
$$

so by (C13)

$$
1 / 600>f_{j}\left(.12391+F_{64}\right),
$$

or

$$
f_{j}<.00229 \quad \text { implying } \quad j \geq 91
$$

so by (C13)

or

$$
1 / 600>f_{j}\left(.12391+F_{91}\right)
$$

$$
f_{j}<.00208
$$

implying $j \geq 99$. as asserted in (C11).

To prove the remaining part of (C11), we note that the third condition of case 9, together with (C4),

\begin{tabular}{|c|c|c|c|c|c|c|c|}
\hline$j$ & $k$ & $u$ & $\Delta C$ & $j$ & $k$ & $u$ & $\Delta C$ \\
\hline 20 & 246 & 0.519 & 466,000 & 114 & 272 & .852 & 367,000 \\
\hline 30 & 253 & .587 & 440,000 & 120 & 264 & .866 & 366,000 \\
\hline 40 & 255 & .642 & 421,000 & 125 & 256 & .878 & 367,000 \\
\hline 50 & 264 & 685 & 407,000 & & & & \\
\hline 60 & 265 & .723 & 398,000 & 130 & 256 & .885 & 367,000 \\
\hline & & & & 135 & 253 & .893 & 369,000 \\
\hline 70 & 265 & .755 & 388,000 & 140 & 252 & .901 & 371,000 \\
\hline 80 & 275 & .778 & 381,000 & 150 & 245 & .919 & 376,000 \\
\hline 90 & 275 & .804 & 375,000 & 160 & 236 & .938 & 388,000 \\
\hline 95 & 275 & .815 & 372,000 & & & & \\
\hline 100 & 275 & .825 & 371,000 & 170 & 226 & .952 & 395,000 \\
\hline & & & & 180 & 213 & $\begin{array}{r}.972 \\
995\end{array}$ & 410,000 \\
\hline 105 & 275 & .835 & 370,000 & 194 & 200 & .995 & 433,000 \\
\hline 110 & 275 & .847 & 368,000 & 197 & 197 & 1. 000 & 440,000 \\
\hline
\end{tabular}
yields

$$
f_{k}\left(2+F_{j}-F_{k}\right) \leq 1 / 600,
$$

and since $j \geq 99$ and $k \leq 281$, we have

yielding:

$$
f_{k}\left(2+F_{99}-F_{281}\right) \leq 1 / 600,
$$

$$
f_{k} \leq .000916
$$

s) that $k \geq 187$ as asserted in (C11).

The 3 cases not treated so far are

$$
\begin{array}{llll}
\text { Case 10: } & j \leq 114, & m>j, & j \leq n \leq k, \\
\text { Case 11: } & j>114, & m \leq j, & n>k, \\
\text { Case 12: } & j>114, & m \leq j, & j \leq n \leq k .
\end{array}
$$

In all these cases, $D=0$ can occur, and no reduction like that in case 9 appeared possible. We therefore resorted to numerical exploration for these three cases and also for the range $(\mathrm{C} 11)$ of case 9 . A number of values of $j$ were used; for each the value of $k$ such that $D=0$ was found and the corresponding value of $\Delta C$ was computed. The results of the calculations are given in the following table, which supplies the conclusions given in (14.23) and (14.24).

Calculations * for $($ C11) and cases 10 to 12

* The quantity $u$ can be interpreted as the ratio of the actual hourly input of the second loading complex of each subsystem to its maximum input $(36,000$ letters $/ \mathrm{hr}$ ).

Washington, D.C.

(Paper 63B2-11) 